\title{
FINITE-TIME BLOW-UP IN A DEGENERATE CHEMOTAXIS SYSTEM WITH FLUX LIMITATION
}

\author{
NICOLA BELLOMO AND MICHAEL WINKLER
}

ABSTRACT. This paper is concerned with radially symmetric solutions of the parabolic-elliptic version of the Keller-Segel system with flux limitation, as given by

$$
\left\{\begin{array}{l}
u_{t}=\nabla \cdot\left(\frac{u \nabla u}{\sqrt{u^{2}+|\nabla u|^{2}}}\right)-\chi \nabla \cdot\left(\frac{u \nabla v}{\sqrt{1+|\nabla v|^{2}}}\right), \\
0=\Delta v-\mu+u,
\end{array}\right.
$$

under the initial condition $\left.u\right|_{t=0}=u_{0}>0$ and no-flux boundary conditions in a ball $\Omega \subset \mathbb{R}^{n}$, where $\chi>0$ and $\mu:=\frac{1}{|\Omega|} \int_{\Omega} u_{0}$. A previous result of the authors [Comm. Partial Differential Equations 42 (2017), 436-473] has asserted global existence of bounded classical solutions for arbitrary positive radial initial data $u_{0} \in C^{3}(\bar{\Omega})$ when either $n \geq 2$ and $\chi<1$, or $n=1$ and $\int_{\Omega} u_{0}<\frac{1}{\sqrt{\left(\chi^{2}-1\right)+}}$.

This present paper shows that these conditions are essentially optimal: Indeed, it is shown that if the taxis coefficient satisfies $\chi>1$, then for any choice of

$$
\begin{cases}m>\frac{1}{\sqrt{\chi^{2}-1}} & \text { if } n=1, \\ m>0 \text { is arbitrary } & \text { if } n \geq 2,\end{cases}
$$

there exist positive initial data $u_{0} \in C^{3}(\bar{\Omega})$ satisfying $\int_{\Omega} u_{0}=m$ which are such that for some $T>0,(\star)$ possesses a uniquely determined classical solution $(u, v)$ in $\Omega \times(0, T)$ blowing up at time $T$ in the sense that $\lim \sup _{t \nearrow T}\|u(\cdot, t)\|_{L^{\infty}(\Omega)}=\infty$.

This result is derived by means of a comparison argument applied to the doubly degenerate scalar parabolic equation satisfied by the mass accumulation function associated with $(\star)$.

\section{INTRODUCTION}

Flux-limited Keller-Segel systems. This paper presents a continuation of the analytical study [8] of a flux-limited chemotaxis model recently derived as a development of the classical pattern formation model proposed by Keller and Segel ([30]) to model collective behavior of populations mediated by a chemoattractant. In a general form, this model describes the spatio-temporal evolution of the cell

Received by the editors January 3, 2017 and, in revised form, March 18, 2017.

2010 Mathematics Subject Classification. Primary 35B44; Secondary 35K65, 92C17.

Key words and phrases. Chemotaxis, flux limitation, blow-up, degenerate diffusion.

The first author acknowledges partial support by the Italian Minister for University and Research, PRIN Project coordinated by M. Pulvirenti.

The second author acknowledges support by the Deutsche Forschungsgemeinschaft in the context of the project Analysis of chemotactic cross-diffusion in complex frameworks. 
density $u=u(x, t)$ and the chemoattractant concentration $v=v(x, t)$ by means of the parabolic system

$$
\left\{\begin{array}{l}
u_{t}=\nabla \cdot\left(D_{u}(u, v) \frac{u \nabla u}{\sqrt{u^{2}+|\nabla u|^{2}}}-S(u, v) \frac{u \nabla v}{\sqrt{1+|\nabla v|^{2}}}\right)+H_{1}(u, v), \\
v_{t}=D_{v} \Delta v+H_{2}(u, v)
\end{array}\right.
$$

where $D_{u}$ and $D_{v}$ denote the respective diffusivity terms, $S$ represents the chemotactic sensitivity, and $H_{1}$ and $H_{2}$ account for mechanisms of proliferation, degradation, and possibly also interaction. In comparison to the original Keller-Segel system, besides including cell diffusivity inhibited at small densities and hence supporting finite propagation speeds, the main innovative aspect in (1.1) apparently consists in the choice of limited-diffusive and cross-diffusive fluxes in the first equation by a dynamics which is sensitive to gradients.

The heuristic interpretation of the flux-limited nonlinearity in the diffusion terms is induced by the ability that living entities, in general self-propelled particles, show to perceive not only local density, but also gradients. This particular feature characterizes cells $([4])$, but also human crowds $([4,[5])$. This special sensitivity can be introduced in the modeling at the microscopic scale, namely at the scale of cells, thus leading to the description of multicellular systems by equations obtained by suitable generalizations of the approach of the mathematical kinetic theory.

The state of the system is, in this approach, defined by a probability one particle distribution function over the microscopic state, which includes position and velocity, of the interacting entities, while cell-cell interactions are modeled by theoretical tools of stochastic game theory. Interactions are nonlinearly additive, generally nonlocal, and can include the aforementioned sensitivity ability. Once the kinetic-type model has been derived, the study developed in [6] has shown that the particular mathematical structure in (1.1) can be derived by asymptotic limits and time-space scaling. The development of these asymptotic limits is inspired by the classical Hilbert method known in the kinetic theory of classical particles ([18]).

The interest in the qualitative analysis of solutions to phenomenologically derived models for taxis processes (25]) has generated a variety of interesting analytical results reviewed in [26] and more recently in [7]. Within this general framework the role of nonlinear diffusion and, specifically, of flux-limited diffusion, has posed some challenging problems at various levels. Experimental activity toward a thorough understanding of this specific type of mechanism is deeply analyzed in [41, while so far the mathematical literature apparently has concentrated on studying such fluxlimited diffusion processes either without any interaction with further processes, or with comparatively mild couplings such as to zero-order source terms e.g. of FisherKPP type; corresponding results on existence and on propagation properties can be found in [1, 2, [3, 14, 15, and [16], for instance (cf. also the survey [13]).

Blow-up in semilinear and quasilinear chemotaxis systems. The goal of the present work is to clarify to what extent the introduction of such flux limitations may suppress phenomena of blow-up, as known to constitute one of the most striking characteristic features of the classical Keller-Segel system

$$
\left\{\begin{array}{l}
u_{t}=\Delta u-\nabla \cdot(u \nabla v), \\
v_{t}=\Delta v-v+u
\end{array}\right.
$$


and also of several among its derivatives. Indeed, the Neumann initial-boundary value problem for (1.2) is known to possess solutions blowing up in finite time with respect to the spatial $L^{\infty}$ norm of $u$ when either the spatial dimension $n$ satisfies $n \geq 3$ (45), or when $n=2$ and the initially present - and thereafter conservedtotal mass $\int u(\cdot, 0)$ of cells is suitably large $([24$, [34]). On the other hand, in the case $n=2$ appropriately small values of $\int u(\cdot, 0)$ warrant global existence of bounded solutions [36, whereas if $n \geq 3$, then global bounded solutions exist under alternative smallness conditions involving the norms of $(u(\cdot, 0), v(\cdot, 0))$ in $L^{\frac{n}{2}} \times W^{1, n}$ ([17, 42]). In the associated spatially one-dimensional problem, global bounded solutions exist for all reasonably regular initial data, thus reflecting absence of any blow-up phenomenon in this case ([38]).

The knowledge on corresponding features of quasilinear relatives of (1.2) seems most developed for models involving density-dependent variants in the diffusivity and the chemotactic sensitivity. For instance, if $D_{u}$ and $S$ are smooth positive functions on $[0, \infty)$, then the Neumann problem for

$$
\left\{\begin{array}{l}
u_{t}=\nabla \cdot\left(D_{u}(u) \nabla u\right)-\nabla \cdot(S(u) \nabla v), \\
v_{t}=\Delta v-v+u
\end{array}\right.
$$

possesses some unbounded solutions whenever $\frac{S(u)}{D_{u}(u)} \geq C u^{\frac{2}{n}+\varepsilon}$ for all $u \geq 1$ and some $C>0$ and $\varepsilon>0$ ([44]). Beyond this, refined studies have given additional conditions on $D_{u}$ and $S$ under which this singularity formation must occur within finite time, and have moreover identified some particular cases of essentially algebraic behavior of both $D_{u}$ and $S$ in which these explosions must occur in infinite time only (20], 21], 22]; see also [19] for a related example on finite-time blow-up). The optimality of the above growth condition is indicated by a result in [40. and 28. asserting global existence of bounded solutions in the case when $\frac{S(u)}{D_{u}(u)} \leq C u^{\frac{2}{n}-\varepsilon}$ for $u \geq 1$ with some $C>0$ and $\varepsilon>0$, provided that $D_{u}$ decays at most algebraically as $u \rightarrow \infty$ (cf. e.g. [32, [27], [46], and [39] for some among the numerous precedents in this direction).

Besides this, a considerable literature has identified several additional mechanisms as capable of suppressing explosions in Keller-Segel-type systems. These may consist of certain saturation effects in the signal production process at large densities ([33], [11]) or in further dissipation due to superlinear death effects in frameworks of logistic-type cell proliferation ([37, [43]), for instance. A recent deep result has revealed that even the mere inclusion of transport effects by appropriately constructed incompressible vector fields can prevent blow-up in otherwise essentially unchanged Keller-Segel systems in spatially two- and three-dimensional settings $([31])$.

As compared to this, the literature on variants of (1.2) involving modifications of the dependence of fluxes on gradients seems quite thin. Moreover, the few results available in this direction mainly seem to concentrate on modifications in the cross-diffusive term, essentially guided by the underlying idea to rule out blow-up by suitable regularization of the taxis term in (1.2), as apparently justified in appropriate biological contexts (see the discussion in [25] as well as the analytical findings reported there). In particular, we are not aware of any result detecting an explosion in any such context; this may reflect the evident challenges connected to rigorously proving the occurrence of blow-up in such complex chemotaxis systems. 
Main results: Detecting blow-up under optimal conditions. The present work will reveal that actually also the introduction of flux limitations need not necessarily suppress phenomena of chemotactic collapse in the sense of blow-up. In order to make this manifest in a particular setting, let us concentrate on the case when in (1.1) we have $D_{u} \equiv 1$ and $S \equiv$ const. as well as $H_{1} \equiv 0$, and in order to simplify our analysis let us moreover pass to a parabolic-elliptic simplification thereof, thus focusing on a frequently considered limit case of fast signal diffusion (29]). Here we note that e.g. in the previously discussed situations of (1.2) and (1.3), up to few exceptions ([10]) such parabolic-elliptic variants are known to essentially share the same properties as the respective fully parabolic model with regard to the occurrence of blow-up ([35, [9], [12, 23]).

We shall thus subsequently be concerned with the initial-boundary value problem

$$
\left\{\begin{array}{l}
u_{t}=\nabla \cdot\left(\frac{u \nabla u}{\sqrt{u^{2}+|\nabla u|^{2}}}\right)-\chi \nabla \cdot\left(\frac{u \nabla v}{\sqrt{1+|\nabla v|^{2}}}\right), \quad x \in \Omega, t>0, \\
0=\Delta v-\mu+u, \quad x \in \Omega, t>0, \\
\left(\frac{u \nabla u}{\sqrt{u^{2}+|\nabla u|^{2}}}-\chi \frac{u \nabla v}{\sqrt{1+|\nabla v|^{2}}}\right) \cdot \nu=0, \quad x \in \partial \Omega, t>0, \\
u(x, 0)=u_{0}(x), \quad x \in \Omega,
\end{array}\right.
$$

in a ball $\Omega=B_{R}(0) \subset \mathbb{R}^{n}, n \geq 1$, where $\chi>0$ and the initial data are such that

$$
u_{0} \in C^{3}(\bar{\Omega}) \quad \text { is radially symmetric and positive in } \bar{\Omega} \text { with } \frac{\partial u_{0}}{\partial \nu}=0 \text { on } \partial \Omega,
$$

and where

$$
\mu:=\frac{1}{|\Omega|} \int_{\Omega} u_{0}(x) d x
$$

denotes the spatial average of the latter.

In fact, it has been shown in [8] that this problem is well-posed, locally in time, in the following sense.

Theorem A. Let $n \geq 1, \chi>0$, and $\Omega:=B_{R}(0) \subset \mathbb{R}^{n}$ with some $R>0$, and suppose that $u_{0}$ complies with (1.5). Then there exist $T_{\max } \in(0, \infty]$ and a uniquely determined pair $(u, v)$ of positive radially symmetric functions $u \in C^{2,1}\left(\bar{\Omega} \times\left[0, T_{\max }\right)\right)$ and $v \in C^{2,0}\left(\bar{\Omega} \times\left[0, T_{\max }\right)\right)$ which solve (1.4) classically in $\Omega \times\left(0, T_{\max }\right)$, and which are such that

$$
\text { if } T_{\max }<\infty, \quad \text { then } \quad \limsup _{t \nearrow T_{\max }}\|u(\cdot, t)\|_{L^{\infty}(\Omega)}=\infty .
$$

Now in order to formulate our results and put them in perspective adequately, let us moreover recall the following statement on global existence and boundedness in certain subcritical cases which has been achieved in [8].

Theorem B. Let $\Omega:=B_{R}(0) \subset \mathbb{R}^{n}$ with some $R>0$, and assume that $u_{0}$ satisfies (1.5), and that either

$$
n \geq 2 \quad \text { and } \quad \chi<1,
$$

or

$$
n=1, \quad \chi>0, \quad \text { and } \quad \int_{\Omega} u_{0}<m_{c}
$$


where in the case $n=1$ we have set

$$
m_{c}:= \begin{cases}\frac{1}{\sqrt{\chi^{2}-1}} & \text { if } \chi>1, \\ +\infty & \text { if } \chi \leq 1 .\end{cases}
$$

Then the problem (1.4) possesses a unique global classical solution $(u, v) \in$ $C^{2,1}(\bar{\Omega} \times[0, \infty)) \times C^{2,0}(\bar{\Omega} \times[0, \infty))$ which is radially symmetric and such that for some $C>0$ we have

$$
\|u(\cdot, t)\|_{L^{\infty}(\Omega)} \leq C \quad \text { and } \quad\|v(\cdot, t)\|_{L^{\infty}(\Omega)} \leq C \quad \text { for all } t>0 .
$$

It is the purpose of the present work to complement the above result on global existence by showing that in both cases $n \geq 2$ and $n=1$, the conditions (1.8) and (1.9) are by no means artificial and of purely technical nature, but that in fact they are essentially optimal in the sense that if appropriate reverse inequalities hold, then finite-time blow-up may occur. To be more precise, the main results of this paper can be formulated as follows.

Theorem 1.1. Let $n \geq 1$ and $\Omega:=B_{R}(0) \subset \mathbb{R}^{n}$ with some $R>0$, and suppose that

$$
\chi>1
$$

and that

$$
\begin{cases}m>m_{c} & \text { if } n=1, \\ m>0 \text { is arbitrary } & \text { if } n \geq 2,\end{cases}
$$

where $m_{c}$ is as in (1.10). Then there exists a nondecreasing function $M_{m} \in$ $C^{0}([0, R])$ fulfilling $\sup _{r \in(0, R)} \frac{M_{m}(r)}{\left|B_{r}(0)\right|}<\infty$ and $M_{m}(R) \leq m$, which is such that whenever $u_{0}$ satisfies (1.5) as well as

$$
\int_{B_{r}(0)} u_{0}(x) d x \geq M_{m}(r) \quad \text { for all } r \in[0, R]
$$

the solution $(u, v)$ of (1.4) blows up in finite time in the sense that in Theorem A we have $T_{\max }<\infty$ and

$$
\limsup _{t \nearrow T_{\max }}\|u(\cdot, t)\|_{L^{\infty}(\Omega)}=\infty .
$$

Indeed the set of all initial data leading to explosions in (1.4) is considerably large in that it firstly contains an open subset with respect to the norm in $L^{\infty}(\Omega)$, and that it secondly possesses some density property within the set of all initial data admissible in the sense of (1.5).

Proposition 1.2. Let $n \geq 1, R>0, \Omega:=B_{R}(0) \subset \mathbb{R}^{n}$, and $\chi>1$.

(i) Let $m>0$ satisfy (1.13). Then there exists a radially symmetric positive $u_{m} \in C^{\infty}(\bar{\Omega})$ which is such that $\frac{\partial u_{m}}{\partial \nu}=0$ on $\partial \Omega$ and $\int_{\Omega} u_{m}=m$, and for which it is possible to choose $\varepsilon>0$ with the property that whenever $u_{0}$ satisfies (1.5) as well as $\left\|u_{0}-u_{m}\right\|_{L^{\infty}(\Omega)} \leq \varepsilon$, the corresponding solution of (1.4) blows up in finite time.

(ii) Given any $u_{0}$ fulfilling (1.5), one can find functions $u_{0 k}, k \in \mathbb{N}$, which satisfy (1.5) and $u_{0 k} \rightarrow u_{0}$ in $L^{p}(\Omega)$ as $k \rightarrow \infty$ for all $p \in(0,1)$, and which are such that for all $k \in \mathbb{N}$ the solution of (1.4) emanating from $u_{0 k}$ blows up in finite time. 
In comparison to the classical Keller-Segel system (1.2), these results in particular mean that when $n \geq 2$, the possible occurrence of blow-up does not go along with a critical mass phenomenon, but that there rather exists a critical sensitivity parameter, namely $\chi=1$, which distinguishes between existence and nonexistence of blow-up solutions. On the other hand, if $n=1$, then for any $\chi>1$, beyond this there exists a critical mass phenomenon, in quite the same flavor as present in (1.2) when $n=2$.

Plan of the paper. Due to the apparent lack of an adequate global dissipative structure, a blow-up analysis for (1.4) cannot be built on the investigation of any energy functional, as possible in both the original Keller-Segel system (1.2) and its quasilinear variant (1.3) $(45]$, 34, 20]). Apart from this, any reasoning in this direction needs to adequately cope with the circumstance that as compared to (1.2), in (1.4) the cross-diffusive flux is considerably inhibited wherever $|\nabla v|$ is large, which seems to prevent access to blow-up arguments based on tracking the evolution of weighted $L^{1}$ norms of $u$ such as e.g. the moment-like functionals considered in 35.

That blow-up may occur despite this strong limitation of cross-diffusive flux will rather be shown by a comparison argument. Indeed, it can readily be verified (Lemma 2.1) that given a radial solution $u$ of (1.4) in $B_{R} \times(0, T)$, the mass accumulation function $w=w(s, t)$, as defined in a standard manner by introducing

$$
w(s, t):=\int_{0}^{s^{\frac{1}{n}}} r^{n-1} u(r, t) d r, \quad(s, t) \in\left[0, R^{n}\right] \times[0, T),
$$

satisfies a scalar parabolic equation which is doubly degenerate, both in space as well as with respect to the variable $w_{s}$, but after all allows for an appropriate comparison principle for certain generalized sub- and supersolutions (Lemma 5.1).

Accordingly, at the core of our analysis will be the construction of suitable subsolutions to the respective problem; in fact, we shall find such subsolutions $\underline{w}$ which undergo a finite-time gradient blow-up at the origin in the sense that for some $T>0$ we have $\sup _{s \in\left(0, R^{n}\right)} \frac{\underline{w}(s, t)}{s} \rightarrow \infty$ as $t \nearrow T$, implying blow-up of $u$ before or at time $T$ whenever $w(\cdot, 0)$ lies above $\underline{w}(\cdot, 0)$. These subsolutions will have a composite structure to be described in Lemma 3.2, matching a nonlinear and essentially parabola-like behavior in a small ball around the origin to an affine linear behavior in a corresponding outer annulus, the latter increasing so as to coincide with the whole domain $B_{R}$ at the blow-up time of $\underline{w}$. The technical challenge, to be addressed in Section 3, will then consist of carefully adjusting the parameters in the definition of $\underline{w}$ in such a manner that the resulting function in fact has the desired blow-up property, where the cases $n \geq 2$ and $n=1$ will require partially different arguments (Lemma 3.11 and Lemma 3.12 ). The statement from Theorem 1.1 will thereafter result in Section 4.

\section{A parabolic problem SAtisfied By the MASS ACCUMUlation FUnCtion}

Throughout the rest of the paper, we fix $R>0$ and consider (1.4) in the spatial domain $\Omega:=B_{R}(0) \subset \mathbb{R}^{n}, n \geq 1$. Then following a standard procedure ([29]), given a radially symmetric solution $(u, v)=(u(r, t), v(r, t))$ of (1.4) in $\Omega \times[0, T)$ for 
some $T>0$, we consider the associated mass accumulation function $w$ given by

$$
w(s, t):=\int_{0}^{s^{\frac{1}{n}}} r^{n-1} u(r, t) d r \quad \text { for } s \in\left[0, R^{n}\right] \text { and } t \in[0, T) .
$$

In order to describe a basic property of $w$ naturally inherited from $(u, v)$ through (1.4), let us furthermore introduce the parabolic operator $\mathcal{P}$ formally given by

$$
(\mathcal{P} \tilde{w})(s, t):=\tilde{w}_{t}-n^{2} \cdot \frac{s^{2-\frac{2}{n}} \tilde{w}_{s} \tilde{w}_{s s}}{\sqrt{\tilde{w}_{s}^{2}+n^{2} s^{2-\frac{2}{n}} \tilde{w}_{s s}^{2}}}-n \chi \cdot \frac{\left(\tilde{w}-\frac{\mu}{n} s\right) \cdot \tilde{w}_{s}}{\sqrt{1+s^{\frac{2}{n}-2}\left(\tilde{w}-\frac{\mu}{n} s\right)^{2}}} .
$$

We note here that for $T>0$, the above expression $\mathcal{P} \tilde{w}$ is indeed well-defined for all $t \in(0, T)$ and a.e. $s \in\left(0, R^{n}\right)$ if, for instance, $\tilde{w} \in C^{1}\left(\left(0, R^{n}\right) \times(0, T)\right)$ is such that $w_{s}>0$ throughout $\left(0, R^{n}\right) \times(0, T)$ and $\tilde{w}(\cdot, t) \in W^{2, \infty}\left(\left(0, R^{n}\right)\right)$ for all $t \in(0, T)$.

Now the function $w$ in (2.1), which clearly complies with these requirements due to smoothness and positivity of $u$, in fact solves an appropriate initial-boundary value problem associated with $\mathcal{P}$ :

Lemma 2.1. Let $n \geq 1$ and $\chi>0$, and suppose that $(u, v)$ is a positive radially symmetric classical solution of (1.4) in $\Omega \times(0, T)$ for some $T>0$ and some nonnegative radially symmetric $u_{0} \in C^{0}(\bar{\Omega})$. Then the function $w$ defined in (2.1) satisfies

$$
\left\{\begin{array}{l}
(\mathcal{P} w)(s, t)=0, \quad s \in\left(0, R^{n}\right), \quad t \in(0, T), \\
w(0, t)=0, \quad w\left(R^{n}, t\right)=\frac{m}{\omega_{n}}, \quad t \in(0, T), \\
w(s, 0)=\int_{0}^{s^{\frac{1}{n}}} r^{n-1} u_{0}(r) d r, \quad s \in\left[0, R^{n}\right],
\end{array}\right.
$$

where $m:=\int_{\Omega} u_{0}(x) d x$ and where $\omega_{n}$ denotes the $(n-1)$-dimensional measure of the unit sphere in $\mathbb{R}^{n}$.

Proof. Omitting the arguments $r, t$ and $s:=r^{n}$ in expressions like $u(r, t)$ and $w(s, t)$, upon an integration in the radial version of the first equation in (1.4) we obtain

$$
\begin{aligned}
w_{t} & =\int_{0}^{s^{\frac{1}{n}}} r^{n-1} u_{t}(r, t) d r \\
& =\int_{0}^{s^{\frac{1}{n}}}\left\{\left(r^{n-1} \frac{u u_{r}}{\sqrt{u^{2}+u_{r}^{2}}}\right)_{r}-\chi\left(r^{n-1} \frac{u v_{r}}{\sqrt{1+v_{r}^{2}}}\right)_{r}\right\} d r \\
& =\left(s^{\frac{1}{n}}\right)^{n-1} \cdot \frac{u u_{r}}{\sqrt{u^{2}+u_{r}^{2}}}-\chi \cdot \frac{u \cdot\left(r^{n-1} v_{r}\right)}{\sqrt{1+v_{r}^{2}}}
\end{aligned}
$$

for $s \in\left(0, R^{n}\right)$ and $t \in(0, T)$. Here in order to replace $v_{r}$, we integrate the second equation in (1.4), that is, the identity $\left(r^{n-1} v_{r}\right)_{r}=\mu r^{n-1}-r^{n-1} u$, to see that

$$
r^{n-1} v_{r}=\frac{\mu}{n} \cdot r^{n}-\int_{0}^{r} \rho^{n-1} u(\rho, t) d \rho=\frac{\mu}{n} \cdot s-w .
$$

Furthermore (2.1) can be used to derive

$$
u=n w_{s} \quad \text { and } \quad u_{r}=n^{2} s^{1-\frac{1}{n}} w_{s s},
$$


to infer from (2.4) that

$$
\begin{aligned}
w_{t} & =s^{1-\frac{1}{n}} \cdot \frac{n w_{s} \cdot n^{2} s^{1-\frac{2}{n}} w_{s s}}{\sqrt{n^{2} w_{s}^{2}+n^{4} s^{2-\frac{2}{n}} w_{s s}^{2}}}-\chi \cdot \frac{n w_{s} \cdot\left(\frac{\mu}{n} s-w\right)}{\sqrt{1+\left(\frac{\mu}{n} s^{\frac{1}{n}}-s^{\frac{1}{n}-1} w\right)^{2}}} \\
& =n^{2} \cdot \frac{s^{2-\frac{2}{n}} w_{s} w_{s s}}{\sqrt{w_{s}^{2}+n^{2} s^{2-\frac{2}{n}} w_{s s}^{2}}}+n \chi \cdot \frac{\left(w-\frac{\mu}{n} s\right) \cdot w_{s}}{\sqrt{1+\left(s^{\frac{1}{n}-1} w-\frac{\mu}{n} s^{\frac{1}{n}}\right)^{2}}}
\end{aligned}
$$

for $s \in\left(0, R^{n}\right)$ and $t \in(0, T)$. This proves the parabolic equation in (2.3), whereas the statements therein concerning boundary and initial conditions can easily be checked using (2.1) and the mass conservation property $\int_{\Omega} u(x, t) d x=\int_{\Omega} u_{0}(x) d x=$ $m$ for $t \in(0, T)$.

\section{Construction of subsolutions for (2.3)}

The goal of this section is to construct subsolutions $\underline{w}$ for the parabolic operator introduced in (2.2) which after some finite time $T$ exhibit a phenomenon of gradient blow-up in the strong sense that

$$
\sup _{s \in\left(0, R^{n}\right)} \frac{\underline{w}(s, t)}{s} \rightarrow+\infty \quad \text { as } t \nearrow T .
$$

Since by means of a suitable comparison principle (cf. Lemma 5.1 in the appendix) we will be able to assert that $w \geq \underline{w}$ in $\left[0, R^{n}\right] \times[0, T)$, this will entail a similar conclusion for $w$ and hence prove that $u$ cannot exist as a bounded solution in $\bar{\Omega} \times[0, T]$.

Our comparison functions will be chosen from a family of explicitly given functions $\underline{w}$, the general form of which will be described in Section 3.1. These functions will exhibit a two-component coarse structure, as reflected in substantially different definitions in a temporally shrinking inner region near the spatial origin, and a corresponding outer part. According to a further fine structure in the inner subdomain, our verification of the desired subsolution properties will be split into three parts, to be detailed in Section 3.2, Section 3.3, and Section 3.4, respectively.

3.1. Constructing a family of candidates. Our construction will involve several parameters. The first of these is a number $\lambda \in(0,1)$ which eventually, as we shall see later, can be chosen arbitrarily when $n \geq 2$ (see Lemma 3.12), but needs to be fixed appropriately close to 1 in the case $n=1$, depending on the size of the mass $m=\int_{\Omega} u_{0}$ (Lemma 3.11). Leaving this final choice open at this point, given any $\lambda \in(0,1)$ we abbreviate

$$
a_{\lambda}:=\frac{(1-\lambda)^{2}}{2 \lambda} \quad \text { and } \quad b_{\lambda}:=\frac{3 \lambda-1}{2 \lambda}
$$

and introduce

$$
\varphi(\xi):= \begin{cases}\lambda \xi^{2} & \text { if } \xi \in[0,1] \\ 1-\frac{a_{\lambda}}{\xi-b_{\lambda}} & \text { if } \xi>1 .\end{cases}
$$


It can then easily be verified that $\varphi$ belongs to $C^{1}([0, \infty)) \cap W^{2, \infty}((0, \infty)) \cap$ $C^{2}([0, \infty) \backslash\{1\})$ with

$$
\varphi^{\prime}(\xi)= \begin{cases}2 \lambda \xi & \text { if } \xi \in[0,1), \\ \frac{a_{\lambda}}{\left(\xi-b_{\lambda}\right)^{2}} & \text { if } \xi>1,\end{cases}
$$

and

$$
\varphi^{\prime \prime}(\xi)= \begin{cases}2 \lambda & \text { if } \xi \in[0,1), \\ -\frac{2 a_{\lambda}}{\left(\xi-b_{\lambda}\right)^{3}} & \text { if } \xi>1,\end{cases}
$$

whence in particular $\varphi^{\prime}(\xi)>0$ for all $\xi \geq 0$.

Let us furthermore introduce a collection of time-dependent parameter functions which play a crucial role throughout the rest of the paper.

Lemma 3.1. Let $n \geq 1, m>0, \lambda \in(0,1), K>1, T>0$, and $B \in C^{1}([0, T))$ be such that $B(t) \in(0,1)$ and $K \sqrt{B(t)}<R^{n}$ for all $t \in[0, T)$, and that moreover

$$
B(t) \leq \frac{K^{2}}{4\left(a_{\lambda}+b_{\lambda}\right)^{2}} \quad \text { for all } t \in[0, T),
$$

where $a_{\lambda}$ and $b_{\lambda}$ are as in (3.1). Then

$$
A(t):=\frac{m}{\omega_{n}} \cdot \frac{K^{2}-2 b_{\lambda} K \sqrt{B(t)}+b_{\lambda}^{2} B(t)}{N(t)}, \quad t \in[0, T)
$$

as well as

$$
D(t):=\frac{m}{\omega_{n}} \cdot \frac{a_{\lambda}}{N(t)}, \quad t \in[0, T),
$$

and

$$
E(t):=\frac{m}{\omega_{n}}-R^{n} D(t) \equiv \frac{m}{\omega_{n}} \cdot \frac{K^{2}-\left(a_{\lambda}+b_{\lambda}\right)\left(2 K \sqrt{B(t)}-b_{\lambda} B(t)\right)}{N(t)}
$$

for $t \in[0, T)$, with

$$
N(t):=K^{2}+a_{\lambda} R^{n}-2\left(a_{\lambda}+b_{\lambda}\right)\left(2 K \sqrt{B(t)}-b_{\lambda} B(t)\right), \quad t \in[0, T),
$$

are all well-defined, and we have

$$
A^{\prime}(t)=\frac{m}{\omega_{n}} \cdot \frac{\left(\frac{K}{\sqrt{B(t)}}-b_{\lambda}\right) \cdot\left(a_{\lambda} K^{2}-a_{\lambda} b_{\lambda} R^{n}\right) \cdot B^{\prime}(t)}{N^{2}(t)}
$$

and

$$
D^{\prime}(t)=\frac{m}{\omega_{n}} \cdot \frac{a_{\lambda}\left(a_{\lambda}+b_{\lambda}\right) \cdot\left(\frac{K}{\sqrt{B(t)}}-b_{\lambda}\right) \cdot B^{\prime}(t)}{N^{2}(t)}
$$

for all $t \in(0, T)$.

Proof. Firstly, thanks to (3.5) we have

$$
\frac{2\left(a_{\lambda}+b_{\lambda}\right)}{K} \sqrt{B(t)} \leq 1 \quad \text { for all } t \in[0, T)
$$


which in particular guarantees that the denominators in (3.6), (3.7), and (3.8) are all positive and hence all these functions are well-defined throughout $\left[0, R^{n}\right] \times[0, T)$. Moreover, differentiating in (3.6) we can compute

$$
\begin{aligned}
& \frac{\omega_{n}}{m} \cdot N^{2}(t) A^{\prime}(t) \\
& =\left\{-\frac{b_{\lambda} K B^{\prime}(t)}{\sqrt{B(t)}}+b_{\lambda}^{2} B^{\prime}(t)\right\} \\
& \times\left\{K^{2}+a_{\lambda} R^{n}-\left(a_{\lambda}+b_{\lambda}\right)\left(2 K \sqrt{B(t)}-b_{\lambda} B(t)\right)\right\} \\
& -\left\{K^{2}+2 b_{\lambda} K \sqrt{B(t)}+b_{\lambda}^{2} B(t)\right\} \\
& \times\left\{-\frac{\left(a_{\lambda}+b_{\lambda}\right) K B^{\prime}(t)}{\sqrt{B(t)}}+\left(a_{\lambda}+b_{\lambda}\right) b_{\lambda} B^{\prime}(t)\right\} \\
& =B^{\prime}(t) \cdot\left\{\frac{K}{\sqrt{B(t)}}-b_{\lambda}\right\} \\
& \times\left\{-b_{\lambda} \cdot\left\{K^{2}+a_{\lambda} R^{n}-d\left(a_{\lambda}+b_{\lambda}\right)\left(2 K \sqrt{B(t)}-b_{\lambda} B(t)\right)\right\}\right. \\
& \left.+\left(a_{\lambda}+b_{\lambda}\right) \cdot\left\{K^{2}-2 b_{\lambda} K \sqrt{B(t)}+b_{\lambda}^{2} B(t)\right\}\right\} \\
& =B^{\prime}(t) \cdot\left\{\frac{K}{\sqrt{B(t)}}-b_{\lambda}\right\} \cdot\left\{a_{\lambda} K^{2}-a_{\lambda} b_{\lambda} R^{n}\right\} \quad \text { for all } t \in(0, T) \text {, }
\end{aligned}
$$

which establishes (3.10). Similarly, differentiation in (3.7) readily yields (3.11).

With these definitions, we can now specify the basic structure of our comparison functions $\underline{w}$ to be used in the sequel. Here a second parameter $K$ enters, to be chosen suitably large finally, as well as a parameter function $B$ depending on time. In combination, these two ingredients determine a line $s=K \sqrt{B(t)}$ in the $(s, t)$-plane which will separate an inner from an outer region and thereby imply a composite structure of $\underline{w}$ as follows.

Lemma 3.2. Let $n \geq 1, m>0, \lambda \in(0,1)$, and $K>1$, and suppose that $T>0$ and that $B \in C^{1}([0, T))$ is such that (3.5) holds as well as $B(t) \in(0,1)$ and $K \sqrt{B(t)}<R^{n}$ for all $t \in[0, T)$. Let

$$
\underline{w}(s, t):= \begin{cases}w_{\text {in }}(s, t) & \text { if } t \in[0, T) \text { and } s \in[0, K \sqrt{B(t)}], \\ w_{\text {out }}(s, t) & \text { if } t \in[0, T) \text { and } s \in\left(K \sqrt{B(t)}, R^{n}\right],\end{cases}
$$

where

$$
w_{i n}(s, t):=A(t) \varphi(\xi), \quad \xi=\xi(s, t):=\frac{s}{B(t)}, \quad t \in[0, T), s \in[0, K \sqrt{B(t)}],
$$


with $\varphi$ as in (3.2) and $A$ as in (3.6), and where

$$
w_{\text {out }}(s, t):=D(t) s+E(t) \quad \text { for } t \in[0, T) \text { and } s \in\left(K \sqrt{B(t)}, R^{n}\right],
$$

with $D$ and $E$ taken from (3.7) and (3.8).

Then $\underline{w}$ is well-defined and continuously differentiable in $\left[0, R^{n}\right] \times[0, T)$ and in addition satisfies $\underline{w}(\cdot, t) \in W^{2, \infty}\left(\left(0, R^{n}\right)\right) \cap C^{2}\left(\left[0, R^{n}\right] \backslash\{B(t), K \sqrt{B(t)}\}\right)$ for all $t \in[0, T)$ as well as

$$
w(0, t)=0 \quad \text { and } \quad w\left(R^{n}, t\right)=\frac{m}{\omega_{n}} \quad \text { for all } t \in(0, T) .
$$

Proof. We first note that for each $t \in[0, T)$, both intervals $[0, K \sqrt{B(t)}]$ and $\left(K \sqrt{B(t)}, R^{n}\right]$ in (3.12) are not empty due to our assumptions that $B(t)>0$ and $K \sqrt{B(t)}<R^{n}$, and that furthermore both statements in (3.15) are direct consequences of (3.8) and the fact that $\varphi(0)=0$ according to (3.2).

To establish the claimed regularity properties of $\underline{w}$, in view of the above observation that $\varphi \in C^{1}([0, \infty)) \cap W^{2, \infty}((0, \infty)) \cap C^{2}([0, \infty) \backslash\{1\})$ we only need to make sure that $\underline{w}, \underline{w}_{s}$, and $\underline{w}_{t}$ are continuous along the line where $s=K \sqrt{B(t)}$, which amounts to showing that

$$
A(t) \cdot \varphi\left(\frac{K}{\sqrt{B(t)}}\right)=D(t) \cdot K \sqrt{B(t)}+E(t) \quad \text { for all } t \in[0, T)
$$

and

$$
\frac{A(t)}{B(t)} \cdot \varphi^{\prime}\left(\frac{K}{\sqrt{B(t)}}\right)=D(t) \quad \text { for all } t \in[0, T)
$$

as well as

$$
A^{\prime}(t) \cdot \varphi\left(\frac{K}{\sqrt{B(t)}}\right)-\frac{K A(t) B^{\prime}(t)}{\sqrt{B(t)}^{3}} \cdot \varphi^{\prime}\left(\frac{K}{\sqrt{B(t)}}\right)=D^{\prime}(t) \cdot K \sqrt{B(t)}+E^{\prime}(t)
$$

for all $t \in[0, T)$.

To derive (3.16), we use (3.2) to see that

$$
\begin{aligned}
A(t) \cdot \varphi\left(\frac{K}{\sqrt{B(t)}}\right)+\left(R^{n}-K \sqrt{B(t)}\right) \cdot D(t) \\
=\frac{m}{\omega_{n}} \cdot \frac{\left(K-b_{\lambda} \sqrt{B(t)}\right)^{2}}{K^{2}+a_{\lambda} R^{n}-\left(a_{\lambda}+b_{\lambda}\right)\left(2 K \sqrt{B(t)}-b_{\lambda} B(t)\right)} \cdot \frac{\frac{K}{\sqrt{B(t)}}-a_{\lambda}-b_{\lambda}}{\frac{K}{\sqrt{B(t)}}-b_{\lambda}} \\
+\left(R^{n}-K \sqrt{B(t)}\right) \cdot \frac{m}{\omega_{n}} \cdot \frac{a}{K^{2}+a_{\lambda} R^{n}-\left(a_{\lambda}+b_{\lambda}\right)\left(2 K \sqrt{B(t)}-b_{\lambda} B(t)\right)} \\
=\frac{m}{\omega_{n}} \cdot \frac{\frac{K-\left(a_{\lambda}+b_{\lambda}\right) \sqrt{B(t)}}{K-b_{\lambda} \sqrt{B(t)}} \cdot\left(K-b_{\lambda} \sqrt{B(t)}\right)^{2}+a_{\lambda}\left(R^{n}-K \sqrt{B(t)}\right)}{K^{2}+a_{\lambda} R^{n}-\left(a_{\lambda}+b_{\lambda}\right)\left(2 K \sqrt{B(t)}-b_{\lambda} B(t)\right)} \\
=\frac{m}{\omega_{n}} \cdot \frac{K^{2}-\left(a_{\lambda}+b_{\lambda}\right)\left(2 K \sqrt{B(t)}-b_{\lambda} B(t)\right)+a_{\lambda} R^{n}}{K^{2}+a_{\lambda} R^{n}-\left(a_{\lambda}+b_{\lambda}\right)\left(2 K \sqrt{B(t)}-b_{\lambda} B(t)\right)} \\
=\frac{m}{\omega_{n}} \quad \text { for all } t \in[0, T),
\end{aligned}
$$


which due to (3.8) means that indeed

$$
\begin{aligned}
A(t) \cdot & \varphi\left(\frac{K}{\sqrt{B(t)}}\right)-D(t) \cdot K \sqrt{B(t)}-E(t) \\
& =A(t) \cdot \varphi\left(\frac{K}{\sqrt{B(t)}}\right)+\left(R^{n}-K \sqrt{B(t)}\right) D(t)-\frac{m}{\omega_{n}} \\
& =0 \quad \text { for all } t \in[0, T) .
\end{aligned}
$$

Next, from (3.7) and (3.6) it immediately follows that

$$
\begin{aligned}
\frac{D(t)}{A(t)} & =\frac{1}{K^{2}} \cdot \frac{a_{\lambda}}{1-\frac{2 b_{\lambda}}{K} \sqrt{B(t)}+\frac{b_{\lambda}^{2}}{K^{2}} B(t)} \\
& =\frac{a_{\lambda}}{K^{2}-2 b_{\lambda} K \sqrt{B(t)}+b_{\lambda}^{2} B(t)} \\
& =\frac{a_{\lambda}}{B(t)\left(\frac{K}{\sqrt{B(t)}}-b_{\lambda}\right)^{2}} \\
& =\frac{1}{B(t)} \cdot \varphi^{\prime}\left(\frac{K}{\sqrt{B(t)}}\right) \quad \text { for all } t \in[0, T),
\end{aligned}
$$

which establishes (3.17).

Finally, in verifying (3.18) we make use of (3.16) and (3.17) as well as (3.6), (3.7), (3.8), (3.10), and (3.11) to see that

$$
\begin{aligned}
A^{\prime}(t) & \varphi\left(\frac{K}{\sqrt{B(t)}}\right)-\frac{K A(t) B^{\prime}(t)}{\sqrt{B(t)}{ }^{3}} \cdot \varphi^{\prime}\left(\frac{K}{\sqrt{B(t)}}\right)-D^{\prime}(t) \cdot K \sqrt{B(t)}+E^{\prime}(t) \\
= & A^{\prime}(t) \cdot \frac{\frac{m}{\omega_{n}}-\left(R^{n}-K \sqrt{B(t)}\right) \cdot D(t)}{A(t)}-\frac{K A(t) B^{\prime}(t)}{\sqrt{B(t)}} \cdot \frac{B(t) D(t)}{A(t)} \\
+ & \left(R^{n}-K \sqrt{B(t)}\right) \cdot D^{\prime}(t) \\
=\frac{m}{\omega_{n}} & \frac{\left(\frac{K}{\sqrt{B(t)}}-b_{\lambda}\right) \cdot\left(a_{\lambda} K^{2}-a_{\lambda} b_{\lambda} R^{n}\right) \cdot B^{\prime}(t)}{N^{2}(t)} \\
& \times\left\{\frac{m}{\omega_{n}} \cdot \frac{K^{2}-2 b_{\lambda} K \sqrt{B(t)}+b_{\lambda}^{2} B(t)}{N(t)}\right\}^{-1} \\
& \times\left\{\frac{m}{\omega_{n}}-\left(R^{n}-K \sqrt{B(t)}\right) \cdot \frac{m}{\omega_{n}} \cdot \frac{a_{\lambda}}{N(t)}\right\} \\
- & \frac{K B^{\prime}(t)}{\sqrt{B(t)}} \cdot \frac{m}{\omega_{n}} \cdot \frac{a_{\lambda}}{N(t)} \\
+ & \left(R^{n}-K \sqrt{B(t)}\right) \cdot \frac{m}{\omega_{n}} \cdot \frac{a_{\lambda}\left(a_{\lambda}+b_{\lambda}\right)\left(\frac{K}{\sqrt{B(t)}}-b_{\lambda}\right) \cdot B^{\prime}(t)}{N^{2}(t)}
\end{aligned}
$$




$$
\begin{aligned}
& =\frac{m}{\omega_{n}} \cdot \frac{\left(\frac{K}{\sqrt{B(t)}}-b_{\lambda}\right) \cdot\left(a_{\lambda} K^{2}-a_{\lambda} b_{\lambda} R^{n}\right) \cdot B^{\prime}(t)}{N^{2}(t)} \\
& \times\left\{\frac{m}{\omega_{n}} \cdot \frac{K^{2}-2 b_{\lambda} K \sqrt{B(t)}+b_{\lambda}^{2} B(t)}{N(t)}\right\}^{-1} \\
& \times\left\{\frac{m}{\omega_{n}}-\left(R^{n}-K \sqrt{B(t)}\right) \cdot \frac{m}{\omega_{n}} \cdot \frac{a_{\lambda}}{N(t)}\right\} \\
& -\frac{K B^{\prime}(t)}{\sqrt{B(t)}} \cdot \frac{m}{\omega_{n}} \cdot \frac{a_{\lambda}}{N(t)} \\
& +\left(R^{n}-K \sqrt{B(t)}\right) \cdot \frac{m}{\omega_{n}} \cdot \frac{a_{\lambda}\left(a_{\lambda}+b_{\lambda}\right)\left(\frac{K}{\sqrt{B(t)}}-b_{\lambda}\right) \cdot B^{\prime}(t)}{N^{2}(t)} \\
& =\frac{m}{\omega_{n}} \cdot \frac{a_{\lambda} B^{\prime}(t)}{\sqrt{B(t)} N^{2}(t)} \cdot\left\{\frac{\left(K-b_{\lambda} \sqrt{B(t)}\right) \cdot\left(K^{2}-b_{\lambda} R^{n}\right)}{(K-b \sqrt{B(t)})^{2}}\right. \\
& \times\left[N(t)-\left(R^{n}-K \sqrt{B(t)}\right) \cdot a_{\lambda}\right] \\
& \left.-K N(t)+\left(a_{\lambda}+b_{\lambda}\right)\left(R^{n}-K \sqrt{B(t)}\right) \cdot\left(K-b_{\lambda} \sqrt{B(t)}\right)\right\} \\
& =\frac{m}{\omega_{n}} \cdot \frac{a_{\lambda} B^{\prime}(t)}{\left(K-b_{\lambda} \sqrt{B(t)}\right) \sqrt{B(t)} N^{2}(t)} \cdot\left\{\left(K^{2}-b_{\lambda} R^{n}\right)\right. \\
& \times\left[K^{2}-\left(a_{\lambda}+2 b_{\lambda}\right) K \sqrt{B(t)}+\left(a_{\lambda}+b_{\lambda}\right) b_{\lambda} B(t)\right] \\
& -K(K-b \sqrt{B(t)}) \cdot\left[K^{2}+a_{\lambda} R^{n}-\left(a_{\lambda}+b_{\lambda}\right)\left(2 K \sqrt{B(t)}-b_{\lambda} B(t)\right)\right] \\
& \left.+\left(a_{\lambda}+b_{\lambda}\right)\left(R^{n}-K \sqrt{B(t)}\right) \cdot\left(K-b_{\lambda} \sqrt{B(t)}\right)^{2}\right\}
\end{aligned}
$$

for all $t \in(0, T)$.

Since it can be checked in a straightforward manner that herein we have

$$
\begin{aligned}
& \left(K^{2}-b_{\lambda} R^{n}\right) \cdot\left[K^{2}-\left(a_{\lambda}+2 b_{\lambda}\right) K \sqrt{B(t)}+\left(a_{\lambda}+b_{\lambda}\right) b_{\lambda} B(t)\right] \\
& \quad-K(K-b \sqrt{B(t)}) \cdot\left[K^{2}+a_{\lambda} R^{n}-\left(a_{\lambda}+b_{\lambda}\right)\left(2 K \sqrt{B(t)}-b_{\lambda} B(t)\right)\right] \\
& \quad+\left(a_{\lambda}+b_{\lambda}\right)\left(R^{n}-K \sqrt{B(t)}\right) \cdot\left(K-b_{\lambda} \sqrt{B(t)}\right)^{2}=0
\end{aligned}
$$

this shows (3.18) and thereby completes the proof. 
3.2. Subsolution properties: Outer region. Let us first make sure that if the function $B$ entering the above definition of $\underline{w}$ is suitably small and satisfies an appropriate differential inequality, then $\underline{w}$ becomes a subsolution in the corresponding outer region addressed in (3.12).

Lemma 3.3. Let $n \geq 1, \chi>0, m>0, \lambda \in(0,1), K>1$, and $B_{0} \in(0,1)$ be such that $K \sqrt{B_{0}}<R^{n}$ and

$$
B_{0} \leq \frac{K^{2}}{16\left(a_{\lambda}+b_{\lambda}\right)^{2}}
$$

with $a_{\lambda}$ and $b_{\lambda}$ given by (3.1). Then if for some $T>0, B \in C^{1}([0, T))$ is positive and nonincreasing and such that

$$
\left\{\begin{array}{l}
B^{\prime}(t) \geq-\frac{n m \chi K}{2\left(a_{\lambda}+b_{\lambda}\right) \omega_{n} R^{n} \sqrt{1+K^{\frac{2}{n}-2} \frac{m^{2}}{\omega_{n}^{2}}}} \cdot B^{1-\frac{1}{2 n}}(t), \quad t \in(0, T), \\
B(0) \leq B_{0}
\end{array}\right.
$$

the function $w_{\text {out }}$ defined in (3.14) satisfies

$$
\left(\mathcal{P} w_{\text {out }}\right)(s, t) \leq 0 \quad \text { for all } t \in(0, T) \text { and } s \in\left(K \sqrt{B(t)}, R^{n}\right)
$$

with $\mathcal{P}$ given by (2.2).

Proof. Again using that $E(t)=\frac{m}{\omega_{n}}-R^{n} D(t)$ for all $t \in(0, T)$ by (3.8), we have

$$
w_{\text {out }}(s, t)=D(t) s+E(t)=\frac{m}{\omega_{n}}-D(t) \cdot\left(R^{n}-s\right)
$$

for all $t \in(0, T)$ and $s \in\left(K \sqrt{B(t)}, R^{n}\right)$. Hence, recalling (3.11) we obtain

$$
\begin{aligned}
\left(w_{\text {out }}\right)_{t}(s, t) & =-D^{\prime}(t) \cdot\left(R^{n}-s\right) \\
& =-\frac{m}{\omega_{n}} \cdot \frac{a_{\lambda}\left(a_{\lambda}+b_{\lambda}\right) \cdot\left\{\frac{K}{\sqrt{B(t)}}-b_{\lambda}\right\}}{N^{2}(t)} \cdot B^{\prime}(t) \cdot\left(R^{n}-s\right)
\end{aligned}
$$

for all $t \in(0, T)$ and $s \in\left(K \sqrt{B(t)}, R^{n}\right)$, where $N(t)$ is as in (3.9) for such $t$. In order to compensate the positive contribution of this term $\left(w_{\text {out }}\right)_{t}$ to $\mathcal{P} w_{\text {out }}$ by a suitably negative impact of the rightmost term

$$
I(s, t):=-n \chi \cdot \frac{\left(w_{\text {out }}-\frac{\mu}{n} s\right) \cdot\left(w_{\text {out }}\right)_{s}}{\sqrt{1+s^{\frac{2}{n}-2}\left(w_{\text {out }}-\frac{\mu}{n} s\right)^{2}}}, \quad t \in(0, T), s \in\left(K \sqrt{B(t)}, R^{n}\right),
$$

in (2.2), we use (3.22) and (3.7) to rewrite

$$
\begin{aligned}
& w_{\text {out }}(s, t)-\frac{\mu}{n} s=\left(\frac{m}{\omega_{n} R^{n}}-D(t)\right) \cdot\left(R^{n}-s\right) \\
= & \frac{m}{\omega_{n} R^{n}} \cdot\left(1-R^{n} \cdot \frac{\omega_{n}}{m} \cdot D(t)\right) \cdot\left(R^{n}-s\right) \\
= & \frac{m}{\omega_{n} R^{n}} \cdot\left(1-\frac{a_{\lambda} R^{n}}{K^{2}+a_{\lambda} R^{n}-\left(a_{\lambda}+b_{\lambda}\right)\left(2 K \sqrt{B(t)}-b_{\lambda} B(t)\right)}\right) \cdot\left(R^{n}-s\right) \\
= & \frac{m}{\omega_{n} R^{n}} \cdot \frac{K^{2}-2\left(a_{\lambda}+b_{\lambda}\right) K \sqrt{B(t)}+\left(a_{\lambda}+b_{\lambda}\right) b_{\lambda} B(t)}{K^{2}+a_{\lambda} R^{n}-\left(a_{\lambda}+b_{\lambda}\right)\left(2 K \sqrt{B(t)}-b_{\lambda} B(t)\right)} \cdot\left(R^{n}-s\right)
\end{aligned}
$$


for $t \in(0, T)$ and $s \in\left(K \sqrt{B(t)}, R^{n}\right)$. As

$$
K^{2}-2\left(a_{\lambda}+b_{\lambda}\right) K \sqrt{B(t)} \geq \frac{1}{2} K^{2} \quad \text { for all } t \in(0, T)
$$

by (3.19), this in particular implies that

$$
0<w_{\text {out }}(s, t)-\frac{\mu}{n} s \leq \frac{m}{\omega_{n} R^{n}} \cdot R^{n}=\frac{m}{\omega_{n}}
$$

and hence

$$
\left(w_{\text {out }}(s, t)-\frac{\mu}{n} s\right)^{2} \leq \frac{m^{2}}{\omega_{n}^{2}} \quad \text { for all } t \in(0, T) \text { and } s \in\left(K \sqrt{B(t)}, R^{n}\right) .
$$

Since moreover $1 \leq B^{\frac{1}{n}-1}(t)$ for all $t \in(0, T)$ due to the fact that $B_{0}<1$, we can thus estimate the denominator in (3.24) in the considered outer region according to

$$
\begin{aligned}
\sqrt{1-s^{\frac{2}{n}-2}\left(w_{\text {out }}(s, t)-\frac{\mu}{n} s\right)^{2}} & \leq \sqrt{B^{\frac{1}{n}-1}(t)+(K \sqrt{B(t)})^{\frac{2}{n}-2} \cdot \frac{m^{2}}{\omega_{n}^{2}}} \\
& =\sqrt{1+K^{\frac{2}{n}-2} \cdot \frac{m^{2}}{\omega_{n}^{2}}} \cdot B^{\frac{1}{2 n}-\frac{1}{2}}(t)
\end{aligned}
$$

for all $t \in(0, T)$ and $s \in\left(K \sqrt{B(t)}, R^{n}\right)$.

Using (3.27) and (3.25) and that

$$
\left(w_{\text {out }}\right)_{s}(s, t)=D(t)=\frac{m}{\omega_{n}} \cdot \frac{a_{\lambda}}{K^{2}+a_{\lambda} R^{n}-\left(a_{\lambda}+b_{\lambda}\right)\left(2 K \sqrt{B(t)}-\left(a_{\lambda}+b_{\lambda}\right) b_{\lambda} B(t)\right)}
$$

for $t \in(0, T)$ and $s \in\left(K \sqrt{B(t)}, R^{n}\right)$, we thereby find that

$$
\begin{aligned}
-I(s, t) \geq n \chi \cdot \frac{\left(w_{\text {out }}(s, t)-\frac{\mu}{n} s\right) \cdot\left(w_{\text {out }}\right)_{s}(s, t)}{\sqrt{1+K^{\frac{2}{n}-2} \cdot \frac{m^{2}}{\omega_{n}^{2}}} \cdot B^{\frac{1}{2 n}-\frac{1}{2}(t)}} \\
=\frac{n \chi}{\sqrt{1+K^{\frac{2}{n}-2} \cdot \frac{m^{2}}{\omega_{n}^{2}}} \cdot B^{\frac{1}{2}-\frac{1}{2 n}}(t)} \\
\quad \times \frac{m}{\omega_{n} R^{n}} \cdot \frac{K^{2}-\left(a_{\lambda}+b_{\lambda}\right)\left(2 K \sqrt{B(t)}-b_{\lambda} B(t)\right)}{K^{2}+a_{\lambda} R^{n}-\left(a_{\lambda}+b_{\lambda}\right)\left(2 K \sqrt{B(t)}-b_{\lambda} B(t)\right)} \\
\quad \times \frac{m}{\omega_{n}} \cdot \frac{a_{\lambda}}{K^{2}+a_{\lambda} R^{n}-\left(a_{\lambda}+b_{\lambda}\right)\left(2 K \sqrt{B(t)}-b_{\lambda} B(t)\right)} \\
=\frac{m}{\omega_{n}} \cdot \frac{a_{\lambda}}{N^{2}(t)} \cdot\left(R^{n}-s\right) \cdot c_{1} \\
\quad \times\left\{K^{2}-\left(a_{\lambda}+b_{\lambda}\right)\left(2 K \sqrt{B(t)}-b_{\lambda} B(t)\right)\right\} \cdot B^{\frac{1}{2}-\frac{1}{2 n}}(t)
\end{aligned}
$$

for all $t \in(0, T)$ and $s \in\left(K \sqrt{B(t)}, R^{n}\right)$, with $N$ as defined in (3.9) and

$$
c_{1}:=\frac{n m \chi}{\omega_{n} R^{n} \cdot \sqrt{1+K^{\frac{2}{n}-2} \cdot \frac{m^{2}}{\omega_{n}^{2}}}} .
$$


Since evidently $\left(w_{\text {out }}\right)_{s s} \equiv 0$, combining this with (3.26) and (3.23) shows that

$$
\begin{array}{r}
\left(\mathcal{P} w_{\text {out }}\right)(s, t) \leq \frac{m}{\omega_{n}} \cdot \frac{a_{\lambda}}{N^{2}(t)} \cdot\left(R^{n}-s\right) \cdot\left\{\left\{-\frac{\left(a_{\lambda}+b_{\lambda}\right) K}{\sqrt{B(t)}}+\left(a_{\lambda}+b_{\lambda}\right) b_{\lambda}\right\} \cdot B^{\prime}(t)\right. \\
\left.-c_{1} \cdot\left\{K^{2}-2\left(a_{\lambda}+b_{\lambda}\right) K \sqrt{B(t)}+\left(a_{\lambda}+b_{\lambda}\right) b_{\lambda} B(t)\right\} \cdot B^{\frac{1}{2}-\frac{1}{2 n}}(t)\right\} \\
\leq \frac{m}{\omega_{n}} \cdot \frac{a_{\lambda}}{N^{2}(t)} \cdot\left(R^{n}-s\right) \cdot\left\{-\frac{\left(a_{\lambda}+b_{\lambda}\right) K}{\sqrt{B(t)}} \cdot B^{\prime}(t)-\frac{c_{1}}{2} K^{2} B^{\frac{1}{2}-\frac{1}{2 n}}(t)\right\}
\end{array}
$$

for all $t \in(0, T)$ and $s \in\left(K \sqrt{B(t)}, R^{n}\right)$, because $\left(a_{\lambda}+b_{\lambda}\right) b_{\lambda} B^{\prime}(t) \leq 0$ for all $t \in(0, T)$. In view of the definition of $c_{1}, 3.20$ warrants that herein

$$
\begin{aligned}
& -\frac{\left(a_{\lambda}+b_{\lambda}\right) K}{\sqrt{B(t)}} \cdot B^{\prime}(t)-\frac{c_{1}}{2} K^{2} B^{\frac{1}{2}-\frac{1}{2 n}}(t) \\
& =\frac{\left(a_{\lambda}+b_{\lambda}\right) K}{\sqrt{B(t)}} \cdot\left\{-B^{\prime}(t)-\frac{c_{1} K}{2\left(a_{\lambda}+b_{\lambda}\right)} \cdot B^{1-\frac{1}{2 n}}(t)\right\} \\
& \leq 0 \quad \text { for all } t \in(0, T) \text { and } s \in\left(K \sqrt{B(t)}, R^{n}\right),
\end{aligned}
$$

so that (3.21) results from (3.28).

3.3. Subsolution properties: Inner region. We proceed to study under which assumptions on the parameters the function $\underline{w}$ defines a subsolution in the corresponding inner domain. To prepare our analysis, let us first compute the action of the operator $\mathcal{P}$ on $\underline{w}$ in the respective region as follows.

Lemma 3.4. Let $n \geq 1, \chi>0, m>0, \lambda \in(0,1), K>1$, and $T>0$, and suppose that $B \in C^{1}([0, T))$ is positive and satisfies (3.5) as well as $K \sqrt{B(t)}<R^{n}$ for all $t \in[0, T)$. Then the function $w_{\text {in }}$ defined in (3.13) has the property that

$$
\left(\mathcal{P} w_{i n}\right)(s, t)=A^{\prime}(t) \varphi(\xi)+\frac{A(t) \varphi^{\prime}(\xi)}{B(t)} \cdot\left\{-\xi B^{\prime}(t)+J_{1}(s, t)+J_{2}(s, t)\right\}
$$

for all $t \in(0, T)$ and $s \in(0, K \sqrt{B(t)}) \backslash\{B(t)\}$, where $\xi=\xi(s, t)=\frac{s}{B(t)}$, $\mathcal{P}$ is as in (2.2), and

$$
J_{1}(s, t):=-n^{2} \cdot \frac{\xi^{2-\frac{2}{n}} \varphi^{\prime \prime}(\xi)}{\sqrt{B^{\frac{4}{n}-2}(t) \varphi^{\prime 2}(\xi)+n^{2} B^{\frac{2}{n}-2}(t) \xi^{2-\frac{2}{n}} \varphi^{\prime \prime 2}(\xi)}}
$$

and

$$
J_{2}(s, t):=-n \chi \cdot \frac{A(t) \varphi(\xi)-\frac{\mu}{n} B(t) \xi}{\sqrt{1+B^{\frac{2}{n}-2}(t) \xi^{\frac{2}{n}}-2} \cdot\left(A(t) \varphi(\xi)-\frac{\mu}{n} B(t) \xi\right)^{2}}
$$

for $t \in(0, T)$ and $s \in(0, K \sqrt{B(t)}) \backslash\{B(t)\}$.

Proof. Since $\xi_{t}=-\frac{s B^{\prime}(t)}{B^{2}(t)}=-\frac{\xi B^{\prime}(t)}{B(t)}$ and $\xi_{s}=\frac{1}{B(t)}$, we can compute

$$
\left(w_{i n}\right)_{t}=A^{\prime}(t) \varphi(\xi)-\frac{A(t) \xi B^{\prime}(t)}{B(t)} \cdot \varphi^{\prime}(\xi)
$$


as well as

$$
\left(w_{i n}\right)_{s}=\frac{A(t)}{B(t)} \cdot \varphi^{\prime}(\xi) \quad \text { and } \quad\left(w_{i n}\right)_{s s}=\frac{A(t)}{B^{2}(t)} \cdot \varphi^{\prime \prime}(\xi)
$$

for all $t \in(0, T)$ and $s \in(0, K \sqrt{B(t)}) \backslash\{B(t)\}$.

Therefore,

$$
\begin{aligned}
& n^{2} \cdot \frac{s^{2-\frac{2}{n}}\left(w_{i n}\right)_{s}\left(w_{i n}\right)_{s s}}{\sqrt{\left(w_{i n}\right)_{s}^{2}+n^{2} s^{2-\frac{2}{n}}\left(w_{i n}\right)_{s s}^{2}}} \\
& =n^{2} \cdot \frac{(B(t) \xi)^{2-\frac{2}{n}} \cdot \frac{A(t)}{B(t)} \varphi^{\prime}(\xi) \cdot \frac{A(t)}{B^{2}(t)} \varphi^{\prime \prime}(\xi)}{\sqrt{\left(\frac{A(t)}{B(t)} \varphi^{\prime}(\xi)\right)^{2}+n^{2}(B(t) \xi)^{2-\frac{2}{n}} \cdot\left(\frac{A(t)}{B^{2}(t)} \varphi^{\prime \prime}(\xi)\right)^{2}}} \\
& =\frac{n^{2} A(t) \varphi^{\prime}(\xi)}{B(t)} \cdot \frac{\xi^{2-\frac{2}{n}} \varphi^{\prime \prime}(\xi)}{\sqrt{B^{\frac{4}{n}-2}(t) \varphi^{\prime 2}(\xi)+n^{2} B^{\frac{2}{n}-2}(t) \xi^{2-\frac{2}{n}} \varphi^{\prime \prime 2}(\xi)}} \\
& =\frac{A(t) \varphi^{\prime}(\xi)}{B(t)} \cdot J_{1}(s, t)
\end{aligned}
$$

and

$$
\begin{aligned}
& n \chi \cdot \frac{\left(w_{i n}-\frac{\mu}{n} s\right)\left(w_{i n}\right)_{s}}{\sqrt{1+s^{\frac{2}{n}-2}\left(w_{i n}-\frac{\mu}{n} s\right)^{2}}} \\
& =n \chi \cdot \frac{\left(A(t) \varphi(\xi)-\frac{\mu}{n} \cdot B(t) \xi\right) \cdot \frac{A(t)}{B(t)} \varphi^{\prime}(\xi)}{\sqrt{1+(B(t) \xi)^{\frac{2}{n}-2} \cdot\left(A(t) \varphi(\xi)-\frac{\mu}{n} \cdot B(t) \xi\right)^{2}}} \\
& =\frac{A(t) \varphi^{\prime}(\xi)}{B(t)} \cdot J_{2}(s, t)
\end{aligned}
$$

for any such $t$ and $s$.

Finally, by definition (2.2) of $\mathcal{P}$, (3.32) (3.34) prove (3.29).

In further examining (3.29), it will be convenient to know that the factor $A$ appearing in (3.13) is nonincreasing with time, meaning that the first summand on the right-hand side in (3.29) will be nonpositive. It is the objective of the following lemma to assert that this can indeed be achieved by choosing the function $B$ to be nonincreasing and appropriately small throughout $[0, T)$.

Lemma 3.5. Let $n \geq 1, m>0, \lambda \in(0,1)$, and $K>1$ be such that $K \geq \sqrt{b_{\lambda} R^{n}}$, and suppose that $B_{0} \in(0,1)$ satisfies

$$
B_{0} \leq \frac{K^{2}}{4\left(a_{\lambda}+b_{\lambda}\right)^{2}}
$$

Then if $T>0$ and $B \in C^{1}([0, T))$ is a positive and nonincreasing function fulfilling $B(0) \leq B_{0}$, for the function $A$ in (3.6) we have

$$
A^{\prime}(t) \leq 0 \quad \text { for all } t \in(0, T) .
$$


In particular,

$$
A(t) \geq A_{T}:=\frac{m}{\omega_{n}} \cdot \frac{1}{1+\frac{a_{\lambda} R^{n}}{K^{2}}} \quad \text { for all } t \in(0, T) .
$$

Proof. We recall that by (3.10), with $N$ given by (3.9) we have

$$
\frac{\omega_{n}}{m} \cdot N^{2}(t) A^{\prime}(t)=\left(\frac{K}{\sqrt{B(t)}}-b_{\lambda}\right) \cdot\left(a_{\lambda} K^{2}-a_{\lambda} b_{\lambda} R^{n}\right) \cdot B^{\prime}(t) \quad \text { for all } t \in(0, T) \text {. }
$$

Here since our assumption (3.35) implies that $B_{0} \leq \frac{K^{2}}{b_{\lambda}^{2}}$, by monotonicity of $B$ we obtain that

$$
\frac{K}{\sqrt{B(t)}}-b_{\lambda} \geq \frac{K}{\sqrt{B_{0}}}-b_{\lambda} \geq 0 \quad \text { for all } t \in(0, T)
$$

whereas the inequality $K \geq \sqrt{b_{\lambda} R^{n}}$ ensures that

$$
a_{\lambda} K^{2}-a_{\lambda} b_{\lambda} R^{n} \geq 0 .
$$

Again using that $B^{\prime} \leq 0$, from (3.38) we thus conclude that (3.36) holds, whereupon (3.37) follows upon taking $t \nearrow T$ in (3.6).

3.4. Subsolution properties: Very inner region. Now in the part very near the origin where $s<B(t)$ and hence $\xi=\frac{s}{B(t)}<1$, the expression $J_{2}$ in (3.29), originating from the chemotactic term in (1.4), need not be positive due to (3.2) and the linear growth of the minuend $\frac{\mu}{n} B(t) \xi$ in the numerator in (3.31). Fortunately, it turns out that the respective unfavorable effect of this to $\mathcal{P} w_{i n}$ in (3.29) can be overbalanced by a suitable contribution of $J_{1}$, which in fact is negative in this region due to the convexity of $\varphi$ on $(0,1)$. Under an additional smallness assumption on $B$, we can indeed achieve the following.

Lemma 3.6. Let $n \geq 1, \chi>0, m>0, \lambda \in(0,1), K>1$, and $B_{0} \in(0,1)$ be such that $K \sqrt{B_{0}}<R^{n}$ and

$$
B_{0} \leq \frac{K^{2}}{4\left(a_{\lambda}+b_{\lambda}\right)^{2}}
$$

as well as

$$
B_{0} \leq\left(\frac{n}{4 \chi \mu}\right)^{n}
$$

Suppose that $T>0$ and that $B \in C^{1}([0, T))$ is a positive and nonincreasing function satisfying

$$
\left\{\begin{array}{l}
B^{\prime}(t) \geq-\frac{n}{4} B^{1-\frac{1}{n}}(t), \quad t \in(0, T), \\
B(0) \leq B_{0}
\end{array}\right.
$$

Then the function $w_{\text {in }}$ defined in (3.13) has the property that

$$
\left(\mathcal{P} w_{\text {in }}\right)(s, t) \leq 0 \quad \text { for all } t \in(0, T) \text { and } s \in(0, B(t)) .
$$


Proof. Writing $\xi=\frac{s}{B(t)}$ for $t \in(0, T)$ and $s \in(0, B(t))$, in (3.29) we can estimate the taxis term from above according to

$$
\begin{aligned}
J_{2}(s, t) & =-n \chi \cdot \frac{A(t) \varphi(\xi)-\frac{\mu}{n} B(t) \xi}{\sqrt{1+B^{\frac{2}{n}-2}(t) \xi^{\frac{2}{n}-2}\left(A(t) \varphi(\xi)-\frac{\mu}{n} B(t) \xi\right)^{2}}} \\
& \leq n \chi \cdot \frac{\frac{\mu}{n} B(t) \xi}{\sqrt{1}} \\
& =\chi \mu B(t) \xi \quad \text { for all } t \in(0, T) \text { and } s \in(0, B(t)) .
\end{aligned}
$$

We next recall that since $\xi \in(0,1)$ whenever $s \in(0, B(t))$, and hence $\varphi^{\prime}(\xi)=2 \lambda \xi$ and $\varphi^{\prime \prime}(\xi)=2 \lambda$, we have

$$
\begin{aligned}
\frac{B^{\frac{4}{n}-2}(t) \varphi^{\prime 2}(\xi)}{B^{\frac{2}{n}-2} \xi^{\frac{2}{n}-2} \varphi^{\prime \prime 2}(\xi)} & =B^{\frac{2}{n}}(t) \cdot \frac{\varphi^{\prime 2}(\xi)}{\xi^{\frac{2}{n}-2} \varphi^{\prime \prime 2}(\xi)} \\
& =B^{\frac{2}{n}}(t) \cdot \frac{4 \lambda^{2} \xi^{2}}{\xi^{\frac{2}{n}-2} \cdot 4 \lambda^{2}} \\
& =B^{\frac{2}{n}}(t) \cdot \xi^{4-\frac{2}{n}} \\
& \leq B^{\frac{2}{n}}(t) \\
& \leq 1 \quad \text { for all } t \in(0, T) \text { and } s \in(0, B(t)),
\end{aligned}
$$

because $B \leq B_{0} \leq 1$ throughout $(0, T)$. Now since $\sqrt{\varphi^{\prime \prime 2}(\xi)}=\varphi^{\prime \prime}(\xi)$ thanks to the convexity of $\varphi$ on $(0,1)$, in (3.30) we therefore find that

$$
\begin{aligned}
& -J_{1}(s, t)=n^{2} \cdot \frac{\xi^{2-\frac{2}{n}} \varphi^{\prime \prime}(\xi)}{\sqrt{B^{\frac{4}{n}-2}(t) \varphi^{\prime 2}(\xi)+n^{2} B^{\frac{2}{n}-2}(t) \xi^{\frac{2}{n}}-2} \varphi^{\prime \prime 2}(\xi)} \\
& \geq n^{2} \cdot \frac{\xi^{2-\frac{2}{n}} \varphi^{\prime \prime}(\xi)}{\sqrt{\left(1+n^{2}\right) B^{\frac{2}{n}-2}(t) \xi^{\frac{2}{n}-2} \varphi^{\prime \prime 2}(\xi)}} \\
& =\frac{n^{2}}{\sqrt{1+n^{2}}} \cdot B^{1-\frac{1}{n}}(t) \xi^{1-\frac{1}{n}} \quad \text { for all } t \in(0, T) \text { and } s \in(0, B(t)) \text {. }
\end{aligned}
$$

As $\sqrt{1+n^{2}} \leq 2 n$ and hence $\frac{n^{2}}{\sqrt{1+n^{2}}} \geq \frac{n}{2}$, due to (3.43) we thereby obtain from (3.29), applying Lemma 3.5 on the basis of (3.39), that

$$
\begin{aligned}
\frac{B(t)}{A(t) \varphi^{\prime}(\xi)} \cdot\left(\mathcal{P} w_{i n}\right)(s, t) & \leq-\xi B^{\prime}(t)-\frac{n}{2} B^{1-\frac{1}{n}}(t) \xi^{1-\frac{1}{n}}+\chi \mu B(t) \xi \\
& =-\xi \cdot\left\{-B^{\prime}(t)-\frac{n}{2} B^{1-\frac{1}{n}}(t) \xi^{-\frac{1}{n}}+\chi \mu B(t)\right\}
\end{aligned}
$$

for all $t \in(0, T)$ and $s \in(0, B(t))$. Here, using that $\xi<1$ implies that $\xi^{-\frac{1}{n}} \geq 1$, and that the restriction (3.40) on $B_{0}$ ensures that

$$
\frac{\chi \mu B(t)}{\frac{n}{4} B^{1-\frac{1}{n}}(t)}=\frac{4 \chi \mu}{n} \cdot B^{\frac{1}{n}}(t) \leq 1,
$$


we see that

$$
\begin{gathered}
-B^{\prime}(t)-\frac{n}{2} B^{1-\frac{1}{n}}(t) \xi^{-\frac{1}{n}}+\chi \mu B(t) \leq-B^{\prime}(t)-\frac{n}{2} B^{1-\frac{1}{n}}(t)+\frac{n}{4} B^{1-\frac{1}{n}}(t) \\
=-B^{\prime}(t)-\frac{n}{4} B^{1-\frac{1}{n}}(t) \quad \text { for all } t \in(0, T) \text { and } s \in(0, B(t)) .
\end{gathered}
$$

As a consequence of (3.41), the claim therefore results from (3.44).

3.5. Subsolution properties: Intermediate region. The crucial part of our analysis will be concerned with the remaining intermediate region, that is, the outer part of the inner domain where $B(t)<s<K \sqrt{B(t)}$. Here the term $J_{1}$ in (3.29), reflecting the diffusion mechanism in (1.4) and thus inhibiting the tendency toward blow-up, can be estimated from above as follows.

Lemma 3.7. Let $n \geq 1, m>0, K>1$, and $T>0$, and suppose that $B \in C^{1}([0, T)$ is positive and such that (3.5) holds as well as $K \sqrt{B(t)}<R^{n}$ for all $t \in[0, T)$. Then writing $\xi=\frac{s}{B(t)}$, for the function $J_{1}$ introduced in (3.30) we have

$$
J_{1}(s, t) \leq n B^{1-\frac{1}{n}}(t) \xi^{1-\frac{1}{n}} \quad \text { for all } t \in(0, T) \text { and } s \in(B(t), K \sqrt{B(t)}) .
$$

Proof. Since $\xi>1$ and hence $\varphi^{\prime \prime}(\xi)<0$ by (3.4), we have $\left|\varphi^{\prime \prime}(\xi)\right|=-\varphi^{\prime \prime}(\xi)$, so that we may use the trivial estimate

$$
B^{\frac{4}{n}-2}(t) \varphi^{\prime 2}(\xi)+n^{2} B^{\frac{2}{n}-2}(t) \xi^{2-\frac{2}{n}} \varphi^{\prime \prime 2}(\xi) \geq n^{2} B^{\frac{2}{n}-2}(t) \xi^{2-\frac{2}{n}} \varphi^{\prime \prime 2}(\xi)
$$

to infer that

$$
\begin{aligned}
J_{1}(s, t) & =n^{2} \cdot \frac{\xi^{2-\frac{2}{n}}\left|\varphi^{\prime \prime}(\xi)\right|}{\sqrt{B^{\frac{4}{n}-2}(t) \varphi^{\prime 2}(\xi)+n^{2} B^{\frac{2}{n}-2}(t) \xi^{2-\frac{2}{n}} \varphi^{\prime \prime 2}(\xi)}} \\
& \leq n^{2} \cdot \frac{\xi^{2-\frac{2}{n}}\left|\varphi^{\prime \prime}(\xi)\right|}{\sqrt{n^{2} B^{\frac{2}{n}-2}(t) \xi^{2-\frac{2}{n}} \varphi^{\prime \prime 2}(\xi)}} \\
& =n B^{1-\frac{1}{n}}(t) \xi^{1-\frac{1}{n}}
\end{aligned}
$$

holds for any such $t$ and $s$, as claimed.

Our goal will accordingly consist of controlling the term $J_{1}$ in (3.29) from above by a suitably negative quantity. As a first step toward this, we shall make sure that in the root appearing in the denominator of (3.31), the second summand essentially dominates the first upon appropriate choices of the parameters.

Lemma 3.8. Let $n \geq 1, m>0, \lambda \in(0,1), K>1$ with $K \geq \sqrt{b_{\lambda} R^{n}}$, and $B_{0} \in(0,1)$ be such that $K \sqrt{B_{0}}<R^{n}$ and

$$
B_{0} \leq \frac{K^{2}}{4\left(a_{\lambda}+b_{\lambda}\right)^{2}} .
$$

Suppose that for some $T>0, B \in C^{1}([0, T))$ is positive and nonincreasing and such that $B(0) \leq B_{0}$. Then writing $\xi=\frac{s}{B(t)}$ for $s \geq 0$ and $t \geq 0$, we have

$$
\frac{1}{A^{2}(t) B^{\frac{2}{n}-2}(t) \xi^{\frac{2}{n}-2} \varphi^{2}(\xi)} \leq \frac{\omega_{n}^{2}}{\lambda^{2} m^{2}} \cdot\left(1+\frac{a_{\lambda} R^{n}}{K^{2}}\right) \cdot K^{2-\frac{2}{n}} B_{0}^{3-\frac{3}{n}},
$$

for all $t \in(0, T)$ and $s \in(B(t), K \sqrt{B(t)})$. 
Proof. Since $K \geq \sqrt{b_{\lambda} R^{n}}$ and (3.46) holds, we know from Lemma 3.5 that $A(t) \geq$ $A_{T}$ for all $t \in(0, T)$ with $A_{T}$ given by (3.37). Moreover, the fact that $\varphi$ is increasing on $[1, \infty)$ allows us to estimate $\varphi(\xi) \geq 1$ for all $t \in(0, T)$ and $s>B(t)$, because for any such $t$ and $s$ we have $\xi>1$. Hence,

$$
\frac{1}{A^{2}(t) B^{\frac{2}{n}-2}(t) \xi^{\frac{2}{n}-2} \varphi^{2}(\xi)} \leq \frac{1}{\lambda^{2} A_{T}^{2}} \cdot B^{2-\frac{2}{n}}(t) \xi^{2-\frac{2}{n}}
$$

for all $t \in(0, T)$ and $s \in(B(t), K \sqrt{B(t)})$.

As $2-\frac{2}{n} \geq 0$, we may use the restriction $\xi<\frac{K}{\sqrt{B(t)}}$ implied by the inequality $s<K \sqrt{B(t)}$ to estimate

$$
\xi^{2-\frac{2}{n}} \leq K^{2-\frac{2}{n}} B^{1-\frac{1}{n}}(t) \quad \text { for all } t \in(0, T) \text { and } s \in(B(t), K \sqrt{B(t)}) .
$$

Therefore, (3.47) is a consequence of (3.48).

In order to prepare an estimate for the numerator in (3.31) from below, let us state and prove the following elementary calculus lemma.

Lemma 3.9. For $\lambda \in(0,1)$, let $a_{\lambda}$ and $b_{\lambda}$ be as defined in (3.1), and let

$$
\psi_{\lambda}(\xi):=\frac{\xi\left(\xi-b_{\lambda}\right)}{\xi-a_{\lambda}-b_{\lambda}} \quad \text { for } \xi \geq 1 .
$$

Then if the numbers $K>1$ and $B \in(0,1)$ satisfy

$$
B \leq \frac{K^{2}}{4\left(a_{\lambda}+b_{\lambda}\right)^{2}}
$$

we have

$$
\psi_{\lambda}(\xi) \leq \max \left\{\frac{1}{\lambda}, \frac{2 K}{\sqrt{B}}\right\} \quad \text { for all } \xi \in\left[1, \frac{K}{\sqrt{B}}\right]
$$

Proof. Differentiation in (3.49) yields

$$
\begin{aligned}
\psi_{\lambda}^{\prime}(\xi) & =\frac{\left(2 \xi-b_{\lambda}\right)\left(\xi-a_{\lambda}-b_{\lambda}\right)-\left(\xi^{2}-b_{\lambda} \xi\right)}{\left(\xi-a_{\lambda}-b_{\lambda}\right)^{2}} \\
& =\frac{\xi^{2}-2\left(a_{\lambda}+b_{\lambda}\right) \xi+\left(a_{\lambda}+b_{\lambda}\right) b_{\lambda}}{\left(\xi-a_{\lambda}-b_{\lambda}\right)^{2}} \quad \text { for all } \xi>1,
\end{aligned}
$$

from which we obtain that

$$
\psi_{\lambda}^{\prime}(\xi)<0 \quad \text { if and only if } \quad \xi \in\left(\xi_{-}, \xi_{+}\right)
$$

where $\xi_{+}$and $\xi_{-}$are given by

$$
\xi_{ \pm}=a_{\lambda}+b_{\lambda} \pm \sqrt{\left(a_{\lambda}+b_{\lambda}\right)^{2}-\left(a_{\lambda}+b_{\lambda}\right) b_{\lambda}} .
$$


Here by (3.1), we recall that $a_{\lambda}+b_{\lambda}=\frac{\lambda+1}{2}$ in computing

$$
\begin{aligned}
\xi_{ \pm} & =\frac{\lambda+1}{2} \pm \sqrt{\left(\frac{\lambda+1}{2}\right)^{2}-\frac{\lambda+1}{2} \cdot \frac{3 \lambda-1}{2 \lambda}} \\
& =\frac{\lambda+1}{2} \pm \sqrt{\frac{(\lambda+1) \cdot[\lambda(\lambda+1)-(3 \lambda-1)]}{4 \lambda}} \\
& =\frac{\lambda+1}{2} \pm \sqrt{\frac{(\lambda+1)(1-\lambda)^{2}}{4 \lambda}} \\
& =\frac{1}{2} \cdot\left\{\lambda+1 \pm(1-\lambda) \cdot \sqrt{\frac{\lambda+1}{\lambda}}\right\} .
\end{aligned}
$$

Hence,

$$
\begin{aligned}
2\left(\xi_{ \pm}-1\right) & =\lambda-1 \pm(1-\lambda) \cdot \sqrt{\frac{\lambda+1}{\lambda}} \\
& =(1-\lambda) \cdot\left(-1 \pm \sqrt{\frac{\lambda+1}{\lambda}}\right),
\end{aligned}
$$

implying that $\xi_{-}<1<\xi_{+}$. Therefore, 3.52 entails that

$$
\psi_{\lambda}(\xi) \leq \max \left\{\psi_{\lambda}(1), \psi_{\lambda}\left(\frac{K}{\sqrt{B}}\right)\right\} \quad \text { for all } \xi \in\left[1, \frac{K}{\sqrt{B}}\right],
$$

where

$$
\psi_{\lambda}(1)=\frac{1-b_{\lambda}}{1-a_{\lambda}-b_{\lambda}}=\frac{1-\frac{3 \lambda-1}{2 \lambda}}{1-\frac{\lambda+1}{2}}=\frac{\frac{1-\lambda}{2 \lambda}}{\frac{1-\lambda}{2}}=\frac{1}{\lambda} .
$$

Since (3.50) ensures that $a_{\lambda}+b_{\lambda} \leq \frac{K}{2 \sqrt{B}}$ and thus

$$
\begin{aligned}
\psi_{\lambda}\left(\frac{K}{\sqrt{B}}\right) & =\frac{\frac{K^{2}}{B}-b_{\lambda} \cdot \frac{K}{\sqrt{B}}}{\frac{K}{\sqrt{B}}-a_{\lambda}-b_{\lambda}} \\
& \leq \frac{\frac{K^{2}}{B}}{\frac{K}{\sqrt{B}}-a_{\lambda}-b_{\lambda}} \\
& \leq \frac{\frac{K^{2}}{B}}{\frac{K}{2 \sqrt{B}}} \\
& =\frac{2 K}{\sqrt{B}},
\end{aligned}
$$

the inequality (3.53) thus yields (3.51).

On the basis of the above lemma, we can indeed achieve that in the numerator in (3.31) the positive summand prevails.

Lemma 3.10. Let $n \geq 1, m>0, \lambda \in(0,1), K>1, \delta \in(0,1)$, and $B_{0} \in(0,1)$ such that $K \sqrt{B_{0}}<R^{n}$ and

$$
B_{0} \leq \frac{K^{2}}{4\left(a_{\lambda}+b_{\lambda}\right)^{2}}
$$

as well as

$$
\frac{\mu}{n A_{T}} \cdot \max \left\{\frac{B_{0}}{\lambda}, 2 K \sqrt{B_{0}}\right\} \leq \delta
$$


with $\mu$ and $A_{T}$ as in (1.6) and (3.37), respectively. Furthermore, let $T>0$ and $B \in C^{1}([0, T))$ be positive and such that

$$
B(t) \leq B_{0} \quad \text { for all } t \in(0, T) .
$$

Then writing $\xi=\frac{s}{B(t)}$, we have

$A(t) \varphi(\xi)-\frac{\mu}{n} B(t) \xi \geq(1-\delta) A(t) \varphi(\xi) \quad$ for all $t \in(0, T)$ and $s \in(B(t), K \sqrt{B(t)})$.

Proof. With $\psi_{\lambda}$ taken from Lemma 3.9. we first observe that

$$
\begin{aligned}
A(t) \varphi(\xi)-\frac{\mu}{n} B(t) \xi & =A(t) \varphi(\xi) \cdot\left\{1-\frac{\mu B(t) \xi}{n A(t)} \cdot \frac{\xi-b_{\lambda}}{\xi-a_{\lambda}-b_{\lambda}}\right\} \\
& =A(t) \varphi(\xi) \cdot\left\{1-\frac{\mu B(t)}{n A(t)} \cdot \psi_{\lambda}(\xi)\right\}
\end{aligned}
$$

for all $t \in(0, T)$ and $s \in(B(t), K \sqrt{B(t)})$. Here thanks to (3.54) and (3.56) we may apply Lemma 3.9, which combined with (3.37) shows that

$$
\begin{aligned}
\frac{\mu B(t)}{n A(t)} \cdot \psi_{\lambda}(\xi) & \leq \frac{\mu B(t)}{n A_{T}} \cdot \max \left\{\frac{1}{\lambda}, \frac{2 K}{\sqrt{B(t)}}\right\} \\
& \leq \frac{\mu}{n A_{t}} \cdot \max \left\{\frac{B_{0}}{\lambda}, 2 K \sqrt{B_{0}}\right\}
\end{aligned}
$$

for all $t \in(0, T)$ and $s \in(B(t), K \sqrt{B(t)})$.

In light of (3.55), the conclusion (3.57) is therefore a consequence of (3.58).

With the above preparations at hand, we can proceed to show that under the assumptions of Theorem 1.1, if $B$ is a suitably small nonincreasing function satisfying an appropriate differential inequality, then $w_{i n}$ indeed becomes a subsolution of (2.3) in the intermediate region where $B(t)<s<K \sqrt{B(t)}$.

We shall first demonstrate this in the spatially one-dimensional case, in which the role of the number $m_{c}$ in (1.10) will become clear through the following lemma.

Lemma 3.11. Let $n=1, \chi>1$, and $m>m_{c}=\frac{1}{\sqrt{\chi^{2}-1}}$. Then there exist $\lambda \in(0,1), K>1, \kappa_{1}>0$, and $B_{01} \in(0,1)$ such that $K \sqrt{B_{01}}<R$, and such that whenever $T>0$ and $B \in C^{1}([0, T))$ is a positive and nonincreasing function fulfilling (3.5) as well as

$$
\left\{\begin{array}{l}
B^{\prime}(t) \geq-\kappa_{1} \sqrt{B(t)}, \quad t \in(0, T), \\
B(0) \leq B_{01}
\end{array}\right.
$$

then for $w_{\text {in }}$ as in (3.13) we have

$$
\left(\mathcal{P} w_{\text {in }}\right)(s, t) \leq 0 \quad \text { for all } t \in(0, T) \text { and } s \in(B(t), K \sqrt{B(t)}) .
$$

Proof. As $m>m_{c}$, we have $\frac{m \chi}{\sqrt{1+m^{2}}}>1$, whence it is possible to fix $\lambda \in(0,1)$ sufficiently close to 1 such that

$$
\frac{m \chi}{\sqrt{\frac{1}{\lambda^{2}}+m^{2}}}>1 .
$$


This in turn allows us to choose some $\delta \in(0,1)$ such that

$$
c_{2}:=\frac{(1-\delta) m \chi}{\sqrt{\frac{1+\delta}{\lambda^{2}}+m^{2}}}-1
$$

is positive. We thereafter pick $K>1$ such that with $a_{\lambda}$ and $b_{\lambda}$ as in (3.1) we have

$$
K \geq \sqrt{b_{\lambda} R}
$$

and

$$
\frac{a_{\lambda} R}{K^{2}} \leq \delta
$$

Finally, we take $B_{01} \in(0,1)$ conveniently small fulfilling $K \sqrt{B_{01}}<R$ and

$$
B_{01} \leq \frac{K^{2}}{4\left(a_{\lambda}+b_{\lambda}\right)^{2}}
$$

as well as

$$
\frac{\mu}{A_{T}} \cdot \max \left\{\frac{B_{01}}{\lambda}, 2 K \sqrt{B_{01}}\right\} \leq \delta
$$

with $A_{T}$ as in (3.37), and let

$$
\kappa_{1}:=\frac{c_{2}}{K} .
$$

Then given any $T>0$ and a positive nonincreasing $B \in C^{1}([0, T))$ satisfying (3.59), from Lemma 3.5 in conjunction with (3.62) we know that $A^{\prime} \leq 0$ on $(0, T)$, so that (3.29) yields

$$
\frac{B(t)}{A(t) \varphi^{\prime}(\xi)} \cdot\left(\mathcal{P} w_{i n}\right)(s, t) \leq-\xi B^{\prime}(t)+J_{1}(s, t)+J_{2}(s, t)
$$

for all $t \in(0, T)$ and $s \in(B(t), K \sqrt{B(t)})$, with $\xi=\frac{s}{B(t)}$ and $J_{1}$ and $J_{2}$ as given by (3.30) and (3.31).

Here, Lemma 3.7 says that

$$
J_{1}(s, t) \leq 1 \quad \text { for all } t \in(0, T) \text { and } s \in(B(t), K \sqrt{B(t)}),
$$

and in order to compensate this positive contribution in (3.67) appropriately, we first invoke Lemma 3.10, which ensures that thanks to (3.64) and (3.65) we have (3.69)

$$
A(t)-\mu B(t) \xi \geq(1-\delta) A(t) \varphi(\xi) \quad \text { for all } t \in(0, T) \text { and } s \in(B(t), K \sqrt{B(t)}) .
$$

In particular, this implies that the expression on the left-hand side herein is nonnegative, so that we can estimate

$$
(A(t) \varphi(\xi)-\mu B(t) \xi)^{2} \leq A^{2}(t) \varphi^{2}(\xi) \quad \text { for all } t \in(0, T) \text { and } s \in(B(t), K \sqrt{B(t)}) \text {. }
$$

Since (3.62) and (3.64) allow for an application of Lemma 3.8 we moreover know that

$$
\begin{aligned}
\frac{1}{A^{2}(t) \varphi^{2}(\xi)} & \leq \frac{1}{\lambda^{2} m^{2}} \cdot\left(1+\frac{a_{\lambda} R}{K^{2}}\right) \\
& \leq \frac{1+\delta}{\lambda^{2} m^{2}} \quad \text { for all } t \in(0, T) \text { and } s \in(B(t), K \sqrt{B(t)})
\end{aligned}
$$


because of (3.63). Combining this with (3.70) shows that in the denominator in the definition (3.31) of $J_{2}$ we have

$$
\begin{aligned}
\sqrt{1+(A(t) \varphi(\xi)-\mu B(t) \xi)^{2}} & \leq \sqrt{\frac{1+\delta}{\lambda^{2} m^{2}} \cdot A^{2}(t) \varphi^{2}(\xi)+A^{2}(t) \varphi^{2}(\xi)} \\
& =\sqrt{\frac{1+\delta}{\lambda^{2} m^{2}}+1} \cdot A(t) \varphi(\xi)
\end{aligned}
$$

for all $t \in(0, T)$ and $s \in(B(t), K \sqrt{B(t)})$. So that by means of (3.69) we can estimate

$$
\begin{aligned}
-J_{2}(s, t) & =\chi \cdot \frac{A(t) \varphi(\xi)-\mu B(t) \xi}{1+(A(t) \varphi(\xi)-\mu B(t) \xi)^{2}} \\
& \geq \chi \cdot \frac{(1-\delta) A(t) \varphi(\xi)}{\sqrt{\frac{1+\delta}{\lambda^{2} m^{2}}+1} \cdot A(t) \varphi(\xi)} \\
& =\frac{(1-\delta) m \chi}{\sqrt{\frac{1+\delta}{\lambda^{2}}+m^{2}}} \quad \text { for all } t \in(0, T) \text { and } s \in(B(t), K \sqrt{B(t)}) .
\end{aligned}
$$

Together with (3.66) and (3.68), in view of the definition (3.61) of $c_{2}$ this implies that

$$
\frac{B(t)}{A(t) \varphi^{\prime}(\xi)} \cdot\left(\mathcal{P} w_{i n}\right)(s, t) \leq-\xi B^{\prime}(t)+1-\frac{(1-\delta) m \chi}{\sqrt{\frac{1+\delta}{\lambda^{2}}+m^{2}}}=-\xi B^{\prime}(t)-c_{2}
$$

$$
\text { for all } t \in(0, T) \text { and } s \in(B(t), K \sqrt{B(t)}) \text {. }
$$

Once more using that in the considered region we have $\xi \leq \frac{K}{\sqrt{B(t)}}$, due to our choice of $\kappa_{1}$ we infer that

$$
\begin{aligned}
-\xi B^{\prime}(t)+c_{2} & =\xi \cdot\left\{-B^{\prime}(t)-\frac{c_{2}}{\xi}\right\} \\
& \leq \xi \cdot\left\{-B^{\prime}(t)-\frac{c_{2} \sqrt{B(t)}}{K}\right\} \\
& =\xi \cdot\left\{-B^{\prime}(t)-\kappa_{1} \sqrt{B(t)}\right\} \\
& \leq 0 \quad \text { for all } t \in(0, T) \text { and } s \in(B(t), K \sqrt{B(t)})
\end{aligned}
$$

because of (3.59), whereby the proof is completed.

In the case $n \geq 2$, we follow the same basic strategy as above, but numerous adaptations are necessary due to the fact that in this case the more involved, and more degenerate, structure of $J_{1}$ and $J_{2}$ in (3.29) allows for choosing actually any positive value of the mass $m$ whenever $\chi>1$.

Lemma 3.12. Let $n \geq 2, \chi>1$, and $m>0$, and let $\lambda \in(0,1)$ be arbitrary. Then there exist $K>1, \kappa_{n}>0$, and $B_{0 n} \in(0,1)$ such that $K \sqrt{B_{0 n}}<R^{n}$, and such that if $T>0$ and $B \in C^{1}([0, T))$ is positive and nonincreasing such that

$$
\left\{\begin{array}{l}
B^{\prime}(t) \geq-\kappa_{n} B^{1-\frac{1}{2 n}}(t), \quad t \in(0, T), \\
B(0) \leq B_{0 n}
\end{array}\right.
$$


then the function $w_{\text {in }}$ defined in (3.14) satisfies

$$
\left(\mathcal{P} w_{\text {in }}\right)(s, t) \leq 0 \quad \text { for all } t \in(0, T) \text { and } s \in(B(t), K \sqrt{B(t)}) .
$$

Proof. We let $a_{\lambda}$ and $b_{\lambda}$ as in (3.1), take any $K>1$ fulfilling

$$
K>\sqrt{b_{\lambda} R^{n}}
$$

and use that $\chi>1$ to pick $\delta \in(0,1)$ suitably small such that

$$
c_{3}:=n \cdot\left\{\frac{(1-\delta) \chi}{\sqrt{1+\delta}}-1\right\}>0
$$

It is the possible to fix $B_{0 n} \in(0,1)$ such that $K \sqrt{B_{0 n}}<R^{n}$ and

$$
B_{0 n} \leq \frac{K^{2}}{4\left(a_{\lambda}+b_{\lambda}\right)^{2}},
$$

such that with $A_{T}$ as in (3.37) we have

$$
\frac{\mu}{n A_{t}} \cdot \max \left\{\frac{B_{0 n}}{\lambda}, 2 K \sqrt{B_{0 n}}\right\} \leq \delta,
$$

and such that

$$
\frac{\omega_{n}}{\lambda^{2} m^{2}} \cdot\left(1+\frac{a_{\lambda} R^{n}}{K^{2}}\right) \cdot K^{2-\frac{2}{n}} B_{0 n}^{3-\frac{3}{n}} \leq \delta,
$$

where we note that in achieving the latter we make use of our assumption that $n \geq 2$. We finally let

$$
\kappa_{n}:=c_{3} K^{-\frac{1}{n}},
$$

and suppose that $T>0$ and that $B \in C^{1}([0, T))$ is positive and nonincreasing and such that (3.71) holds.

Then (3.73) and (3.75) warrant that Lemma 3.5 applies so as to yield that $A^{\prime} \leq 0$ on $(0, T)$, and that hence by (3.29),

$$
\frac{B(t)}{A(t) \varphi^{\prime}(\xi)} \cdot\left(\mathcal{P} w_{i n}\right)(s, t) \leq-\xi B^{\prime}(t)+J_{1}(s, t)+J_{2}(s, t)
$$

for all $t \in(0, T)$ and $s \in(B(t), K \sqrt{B(t)})$, where again $\xi=\frac{s}{B(t)}$, and where $J_{1}$ and $J_{2}$ are as defined in (3.30) and (3.31), respectively. Now thanks to (3.75) and (3.76), Lemma 3.10 shows that

$A(t) \varphi(\xi)-\frac{\mu}{n} B(t) \xi \geq(1-\delta) A(t) \varphi(\xi) \quad$ for all $t \in(0, T)$ and $s \in(B(t), K \sqrt{B(t)})$,

whereas (3.75) allows for invoking Lemma 3.8 to infer from (3.77) that

$$
\begin{aligned}
\frac{1}{A^{2}(t) B^{\frac{2}{n}-2}(t) \xi^{\frac{2}{n}-2} \varphi^{2}(\xi)} & \leq \frac{\omega_{n}^{2}}{\lambda^{2} m^{2}} \cdot\left(1+\frac{a_{\lambda} R^{n}}{K^{2}}\right) \cdot K^{2-\frac{2}{n}} B_{0 n}^{3-\frac{3}{n}} \\
& \leq \delta \quad \text { for all } t \in(0, T) \text { and } s \in(B(t), K \sqrt{B(t)}) .
\end{aligned}
$$


By means of (3.80), (3.81), and the fact that $\delta<1$, we can thus estimate $J_{2}$ according to

$$
\begin{aligned}
& -J_{2}(s, t)=n \chi \cdot \frac{A(t) \varphi(\xi)-\frac{\mu}{n} B(t) \xi}{\sqrt{1+B^{\frac{2}{n}-2}(t) \xi^{\frac{2}{n}}-2}\left(A(t) \varphi(\xi)-\frac{\mu}{n} B(t) \xi\right)^{2}} \\
& \geq n \chi \cdot \frac{(1-\delta) A(t) \varphi(\xi)}{\sqrt{1+B^{\frac{2}{n}-2}(t) \xi^{\frac{2}{n}}-2\left(A(t) \varphi(\xi)-\frac{\mu}{n} B(t) \xi\right)^{2}}} \\
& \geq n \chi \cdot \frac{(1-\delta) A(t) \varphi(\xi)}{\sqrt{1+B^{\frac{2}{n}-2}(t) \xi^{\frac{2}{n}-2} A^{2}(t) \varphi^{2}(\xi)}} \\
& \geq n \chi \cdot \frac{(1-\delta) A(t) \varphi(\xi)}{\sqrt{(\delta+1) \cdot B^{\frac{2}{n}-2}(t) \xi^{\frac{2}{n}-2} A^{2}(t) \varphi^{2}(\xi)}} \\
& =\frac{(1-\delta) n \chi}{\sqrt{1+\delta}} \cdot B^{1-\frac{1}{n}}(t) \xi^{1-\frac{1}{n}}
\end{aligned}
$$

for all $t \in(0, T)$ and $s \in(B(t), K \sqrt{B(t)})$. Since on the other hand

$$
J_{1}(s, t) \leq n B^{1-\frac{1}{n}}(t) \xi^{1-\frac{1}{n}} \quad \text { for all } t \in(0, T) \text { and } s \in(B(t), K \sqrt{B(t)})
$$

due to Lemma 3.10, we therefore conclude from (3.79) that

$$
\begin{aligned}
\frac{B(t)}{A(t) \varphi^{\prime}(\xi)} \cdot\left(\mathcal{P} w_{i n}\right)(s, t) & \leq-\xi B^{\prime}(t)+n B^{1-\frac{1}{n}}(t) \xi^{1-\frac{1}{n}}-\frac{(1-\delta) n \chi}{\sqrt{1+\delta}} \cdot B^{1-\frac{1}{n}}(t) \xi^{1-\frac{1}{n}} \\
& =-\xi B^{\prime}(t)-c_{3} B^{1-\frac{1}{n}}(t) \xi^{1-\frac{1}{n}}
\end{aligned}
$$

for all $t \in(0, T)$ and $s \in(B(t), K \sqrt{B(t)})$. We finally observe that $\xi<\frac{K}{\sqrt{B(t)}}$ whenever $s<K \sqrt{B(t)}$, and that hence by (3.78),

$$
\begin{aligned}
-\xi B^{\prime}(t)-c_{3} B^{1-\frac{1}{n}}(t) \xi^{1-\frac{1}{n}} & =\xi \cdot\left\{-B^{\prime}(t)-c_{3} B^{1-\frac{1}{n}}(t) \xi^{-\frac{1}{n}}\right\} \\
& \leq \xi \cdot\left\{-B^{\prime}(t)-c_{3} B^{1-\frac{1}{n}}(t) \cdot K^{-\frac{1}{n}} B^{\frac{1}{2 n}}(t)\right\} \\
& =\xi \cdot\left\{-B^{\prime}(t)-\kappa_{n} B^{1-\frac{1}{2 n}}(t)\right\}
\end{aligned}
$$

for all $t \in(0, T)$ and $s \in(B(t), K \sqrt{B(t)})$, so that (3.71) and (3.82) guarantee that indeed the claimed inequality (3.72) holds.

\section{Blow-up. Proof of Theorem 1.1}

Now our main result on blow-up of solutions to the original problem can be derived by a combination of Lemma 3.3 with Lemma 3.6 as well Lemma 3.11 and Lemma 3.12 in the cases $n=1$ and $n \geq 2$, respectively, along with a straightforward comparison argument. 
Proof of Theorem 1.1. Thanks to our assumptions (1.12) and (1.13), depending on whether $n=1$ or $n \geq 2$ we may invoke either Lemma 3.11 or Lemma 3.12 to obtain $\lambda \in(0,1), K>0, \kappa_{n}>0$, and $B_{0 n} \in(0,1)$ with the respective properties listed there. We then fix $B_{0} \in\left(0, B_{0 n}\right]$ such that (3.19), (3.39), and (3.40) hold, and thereafter we take some $\kappa \in\left(0, \kappa_{n}\right]$ satisfying

$$
\kappa \leq \frac{n m \chi K}{2\left(a_{\lambda}+b_{\lambda}\right) \omega_{n} R^{n} \sqrt{1+K^{\frac{2}{n}-2} \frac{m^{2}}{\omega_{n}^{2}}}}
$$

as well as

$$
\kappa \leq \frac{n}{4}
$$

In view of Lemma 3.3, Lemma 3.6, Lemma 3.11, and Lemma 3.12, these choices ensure that if we let $B$ denote the solution of

$$
\left\{\begin{array}{l}
B^{\prime}(t)=-\kappa B^{1-\frac{1}{2 n}}(t), \quad t \in(0, T) \\
B(0)=B_{0}
\end{array}\right.
$$

extended up to its extinction time $T \in(0, \infty)$, that is, if we define

$$
B(t):=\left\{B_{0}^{\frac{1}{2 n}}-\frac{\kappa}{2 n} t\right\}^{2 n}, \quad t \in[0, T),
$$

with

$$
T:=\frac{2 n}{\kappa} \cdot B_{0}^{\frac{1}{2 n}}
$$

then the functions $w_{\text {out }}$ and $w_{\text {in }}$ given by (3.14) and (3.13) are well-defined and satisfy

$$
\left(\mathcal{P} w_{\text {out }}\right)(s, t) \leq 0 \quad \text { for all } t \in(0, T) \text { and } s \in\left(K \sqrt{B(t)}, R^{n}\right)
$$

as well as

$$
\left(\mathcal{P} w_{i n}\right)(s, t) \leq 0 \quad \text { for all } t \in(0, T) \text { and } s \in(0, B(t)) \cup(B(t), K \sqrt{B(t)}) .
$$

Here in employing Lemma 3.3 we make use of (4.1), whereas in applying Lemma 3.6 we note that $-\kappa B^{1-\frac{1}{2 n}}(t) \geq-\frac{n}{4} B^{1-\frac{1}{n}}(t)$ for all $t \in(0, T)$ due to (4.2) and the fact that $B(t) \leq B_{0}<1$ for all $t \in(0, T)$. According to (4.6) and (4.7), Lemma 3.2 asserts that

$$
\underline{w}(s, t):= \begin{cases}w_{\text {in }}(s, t) & \text { if } t \in[0, T) \text { and } s \in[0, K \sqrt{B(t)}], \\ w_{\text {out }}(s, t) & \text { if } t \in[0, T) \text { and } s \in\left(K \sqrt{B(t)}, R^{n}\right],\end{cases}
$$

defines a function $\underline{w} \in C^{1}\left(\left[0, R^{n}\right] \times[0, T)\right)$ which satisfies

$$
\underline{w}(\cdot, t) \in C^{2}\left(\left[0, R^{n}\right] \backslash\{B(t), K \sqrt{B(t)}\}\right) \quad \text { for all } t \in[0, T)
$$

as well as

$$
(\mathcal{P} \underline{w})(s, t) \leq 0 \quad \text { for all } t \in[0, T) \text { and } s \in\left(0, R^{n}\right) \backslash\{B(t), K \sqrt{B(t)}\} .
$$

Therefore, if $u_{0}$ satisfies (1.5) and is such that

$$
\int_{B_{r}(0)} u_{0}(x) d x \geq M_{m}(r):=\omega_{n} \underline{w}\left(r^{n}, 0\right) \quad \text { for all } r \in[0, R],
$$

then the solution $w$ of (2.3) defined through (2.1) satisfies

$$
w(s, 0) \geq \underline{w}(s, 0) \quad \text { for all } s \in\left(0, R^{n}\right),
$$


and furthermore it is clear that

$$
w(0, t)=\underline{w}(0, t)=0 \quad \text { and } \quad w\left(R^{n}, t\right)=\underline{w}\left(R^{n}, t\right)=\frac{m}{\omega_{n}} \quad \text { for all } t \in(0, \tilde{T}),
$$

where $\tilde{T}:=\min \left\{T_{\max }, T\right\}$. In order to assert applicability of the comparison principle from Lemma 5.1 below, we abbreviate $\alpha:=2-\frac{2}{n} \geq 0$ and let

$$
\phi\left(s, t, y_{0}, y_{1}, y_{2}\right):=n^{2} \cdot \frac{s^{\alpha} y_{1} y_{2}}{\sqrt{y_{1}^{2}+n^{2} s^{\alpha} y_{2}^{2}}}+n \chi \cdot \frac{\left(y_{0}-\frac{\mu}{n} s\right) y_{1}}{\sqrt{1+s^{-\alpha}\left(y_{0}-\frac{\mu}{n} s\right)^{2}}}
$$

for $\left(s, t, y_{0}, y_{1}, y_{2}\right) \in G:=\left(0, R^{n}\right) \times(0, \infty) \times \mathbb{R} \times(0, \infty) \times \mathbb{R}$, so that $\phi \in C^{1}(G)$ with

$$
\begin{aligned}
\frac{\partial \phi}{\partial y_{2}}\left(s, t, y_{0}, y_{1}, y_{2}\right) & =n^{2} \cdot \frac{s^{\alpha} y_{1}^{3}}{\sqrt{y_{1}^{2}+n^{2} s^{\alpha} y_{2}^{2}}} \\
& \geq 0 \quad \text { for all }\left(s, t, y_{0}, y_{1}, y_{2}\right) \in G
\end{aligned}
$$

and

$$
\frac{\partial \phi}{\partial y_{1}}\left(s, t, y_{0}, y_{1}, y_{2}\right)=n^{4} \cdot \frac{s^{2 \alpha} y_{2}^{3}}{\sqrt{y_{1}^{2}+n^{2} s^{\alpha} y_{2}^{2}}}+n \chi \cdot \frac{y_{0}-\frac{\mu}{n} s}{\sqrt{1+s^{-\alpha}\left(y_{0}-\frac{\mu}{n} s\right)^{2}}}
$$

for all $\left(s, t, y_{0}, y_{1}, y_{2}\right) \in G$, as well as

$$
\frac{\partial \phi}{\partial y_{0}}\left(s, t, y_{0}, y_{1}, y_{2}\right)=n \chi \cdot \frac{y_{1}}{\sqrt{1+s^{-\alpha}\left(y_{0}-\frac{\mu}{n} s\right)^{2}}}
$$

for all $\left(s, t, y_{0}, y_{1}, y_{2}\right) \in G$.

Therefore, we can estimate

$$
\begin{aligned}
\left|\frac{\partial \phi}{\partial y_{1}}\left(s, t, y_{0}, y_{1}, y_{2}\right)\right| & \leq n s^{\frac{\alpha}{2}} \cdot \frac{{\sqrt{n^{2} s^{\alpha} y_{2}^{2}}}^{3}}{{\sqrt{y_{1}^{2}+n^{2} s^{\alpha} y_{2}^{2}}}^{3}}+n \chi s^{\frac{\alpha}{2}} \cdot \frac{\sqrt{s^{-\alpha}\left(y_{0}-\frac{\mu}{n} s\right)^{2}}}{\sqrt{1+s^{-\alpha}\left(y_{0}-\frac{\mu}{n} s\right)^{2}}} \\
& \leq n R^{\frac{2 \alpha}{n}}+n \chi R^{\frac{2 \alpha}{n}} \quad \text { for all }\left(s, t, y_{0}, y_{1}, y_{2}\right) \in G
\end{aligned}
$$

and

$$
\left|\frac{\partial \phi}{\partial y_{0}}\left(s, t, y_{0}, y_{1}, y_{2}\right)\right| \leq n \chi\left|y_{1}\right| \quad \text { for all }\left(s, t, y_{0}, y_{1}, y_{2}\right) \in G .
$$

Since the inequalities (4.10), (4.11), and (4.12) warrant the validity of the hypotheses (5.1), (5.3), and (5.2) of Lemma 5.1, as a consequence of the latter we obtain that

$$
w(s, t) \geq \underline{w}(s, t) \quad \text { for all } s \in\left[0, R^{n}\right] \text { and } t \in[0, \tilde{T}) .
$$

As $w(0, t)=\underline{w}(0, t)=0$ for all $t \in(0, \tilde{T})$, by the mean value theorem this implies that for each $t \in(0, \tilde{T})$ we can find some $\theta(t) \in\left(0, R^{n}\right)$ with the property that $w_{s}(\theta(t), t)=\frac{w(B(t), t)}{B(t)} \geq \frac{\underline{w}(B(t), t)}{B(t)}=\frac{A(t) \varphi(1)}{B(t)}=\lambda \cdot \frac{A(t)}{B(t)} \quad$ for all $t \in(0, \tilde{T})$.

Recalling that $u(r, t)=w_{s}\left(r^{\frac{1}{n}}, t\right)$ for all $r \in(0, R)$ and $t \in\left(0, T_{\max }\right)$, we thereby infer that

$$
\sup _{r \in(0, R)} u(r, t) \geq w_{s}(\theta(t), t)=\lambda \cdot \frac{A(t)}{B(t)} \quad \text { for all } t \in(0, \tilde{T}) .
$$


In view of the fact that $B(t) \rightarrow 0$ as $t \nearrow T$, and that hence $A(t) \rightarrow \frac{m}{\omega_{n}}$ as $t \nearrow T$ according to (3.6), this entails that we necessarily must have $T_{\max } \leq T<\infty$, so that (1.15) becomes a consequence of the extensibility criterion (1.7).

Proof of Proposition 1.2. As a preparation for both parts of the proof, let us fix a nonincreasing $\zeta \in C^{\infty}([0, \infty))$ such that $\zeta \equiv 0$ in $[1, \infty), \zeta>0$ in $\left[0, \frac{1}{2}\right]$, and $\int_{0}^{1} \sigma^{n-1} \zeta(\sigma) d \sigma=1$, and note that then $\zeta \geq c_{4}:=\zeta\left(\frac{1}{2}\right)$ throughout $\left[0, \frac{1}{2}\right]$.

(i) In order to construct $u_{m}$ for $m>0$ fulfilling (1.13), given any such $m$ we pick numbers $m_{0}$ and $m_{1}$ such that $0<m_{0}<m_{1}<m$ and such that if $n=1$ we moreover have $m_{0}>m_{c}$, and thereafter we choose $r_{0} \in(0, R)$ small enough satisfying

$$
\frac{c_{4} m_{1}}{2^{n} n \omega_{n} r_{0}^{n}} \geq 1+\sup _{r \in(0, R)} \frac{M_{m_{0}}(r)}{\left|B_{r}(0)\right|}
$$

where we rely on Theorem 1.1 in observing that the expression on the right-hand side herein indeed is finite. Then with $\delta:=\frac{m-m_{1}}{|\Omega|}$ and $\theta:=\frac{m_{1}}{\omega_{n} r_{0}^{n}}$,

$$
u_{m}(r):=\delta+\theta \cdot \zeta\left(\frac{r}{r_{0}}\right), \quad r \in[0, R],
$$

evidently defines a positive radial function $u_{m} \in C^{\infty}(\bar{\Omega})$ satisfying $\frac{\partial u_{m}}{\partial \nu}=0$ on $\partial \Omega$, and moreover our selection of $\zeta$ warrants that

$$
\int_{\Omega} u_{m}=\delta|\Omega|+\omega_{n} \theta r_{0}^{n} \int_{0}^{\frac{R}{r_{0}}} \sigma^{n-1} \zeta(\sigma) d \sigma=\delta|\Omega|+m_{1}=m
$$

because $\frac{R}{r_{0}}>1$. Apart from that, since $\delta \geq 0$ we similarly see that

$$
f_{B_{r}(0)} u_{m} \geq \frac{m_{1}}{\omega_{n} r^{n}} \int_{0}^{\frac{r}{r_{0}}} \sigma^{n-1} \zeta(\sigma) d \sigma \quad \text { for all } r \in(0, R),
$$

so that in the case $r \leq \frac{r_{0}}{2}$ we can use our definition of $c_{4}$ to estimate

$$
f_{B_{r}(0)} u_{m} \geq \frac{m_{1}}{\omega_{n} r^{n}} \cdot c_{4} \int_{0}^{\frac{r}{r_{0}}} \sigma^{n-1} d \sigma=\frac{c_{4} m_{1}}{n \omega_{n} r_{0}^{n}}
$$

whereas if $\frac{r_{0}}{2}<r<r_{0}$, then

$f_{B_{r}(0)} u_{m} \geq \frac{m_{1}}{\omega_{n} r^{n}} \int_{0}^{\frac{1}{2}} \sigma^{n-1} \zeta(\sigma) d \sigma \geq \frac{c_{4} m_{1}}{\omega_{n} r^{n}} \int_{0}^{\frac{1}{2}} \sigma^{n-1} d \sigma=\frac{c_{4} m_{1}}{2^{n} n \omega_{n} r^{n}} \geq \frac{c_{4} m_{1}}{2^{n} n \omega_{n} r_{0}^{n}}$.

In view of (4.13), we thus conclude that

$$
f_{B_{r}(0)} u_{m} \geq 1+\frac{M_{m_{0}}(r)}{\left|B_{r}(0)\right|} \quad \text { for all } r \in\left(0, r_{0}\right)
$$

while evidently

$$
\int_{B_{r}(0)} u_{m}=\delta\left|B_{r}(0)\right|+m_{1} \geq m_{1} \quad \text { for all } r \in\left[r_{0}, R\right] .
$$

We now let $\varepsilon:=\min \left\{1, \frac{m_{1}-m_{0}}{|\Omega|}\right\}$ and suppose that $u_{0}$ satisfies (1.5) and is such that $\left\|u_{0}-u_{m}\right\|_{L^{\infty}(\Omega)} \leq \varepsilon$. Then from (4.14) we infer that since $\varepsilon \leq 1$,

$$
f_{B_{r}(0)} u_{0} \geq f_{B_{r}(0)} u_{m}-\varepsilon \geq 1+\frac{M_{m_{0}}(r)}{\left|B_{r}(0)\right|}-\varepsilon \geq \frac{M_{m_{0}}(r)}{\left|B_{r}(0)\right|} \quad \text { for all } r \in\left(0, r_{0}\right) \text {, }
$$


and (4.15) guarantees that

$\int_{B_{r}(0)} u_{0} \geq \int_{B_{r}(0)} u_{m}-\varepsilon\left|B_{r}(0)\right| \geq m_{1}-\varepsilon|\Omega| \geq m_{0} \geq M_{m_{0}}(r) \quad$ for all $r \in\left[r_{0}, R\right]$,

since $\varepsilon \leq \frac{m_{1}-m_{0}}{|\Omega|}$ and since $M_{m_{0}}$ is nondecreasing with $M_{m_{0}}(R) \leq m_{0}$ by Theorem 1.1 .

Therefore, $\int_{B_{r}(0)} u_{0} \geq M_{m_{0}}(r)$ for all $r \in[0, R]$, so that Theorem 1.1]asserts that indeed for any such $u_{0}$ the corresponding solution of (1.4) must blow up.

(ii) To verify the claimed density property, we fix an arbitrary $m>0$ fulfilling (1.13), and choose any $\left(r_{k}\right)_{k \in \mathbb{N}} \subset(0, R)$ such that $r_{k} \rightarrow 0$ as $k \rightarrow \infty$. Then taking $\zeta$ as above, for each $k \in \mathbb{N}$ the function $u_{0 k}$ defined by

$$
u_{0 k}(r):=u_{0}(r)+\theta_{k} \cdot \zeta\left(\frac{r}{r_{k}}\right), \quad r \in[0, R],
$$

with $\theta_{k}:=\frac{m}{\omega_{n} r_{k}^{n}}$, evidently satisfies (1.5), and for all $p \in(0,1)$ we have

$$
\left\|u_{0 k}-u_{0}\right\|_{L^{p}(\Omega)}^{p}=\omega_{n} \theta_{k}^{p} r_{k}^{n} \int_{0}^{1} \sigma^{n-1} \zeta^{p}(\sigma) d \sigma \rightarrow 0 \quad \text { as } k \rightarrow \infty
$$

because $\theta_{k}^{p} r_{k}^{n} \rightarrow 0$ as $k \rightarrow \infty$ for any such $p$. In view of Theorem 1.1 for completing the proof it is thus sufficient to make sure that if we fix $k_{0} \in \mathbb{N}$ large enough fulfilling

$$
\frac{c_{4} m}{2^{n} n \omega_{n} r_{k}^{n}} \geq \sup _{r \in(0, R)} \frac{M_{m}(r)}{\left|B_{r}(0)\right|} \quad \text { for all } k \geq k_{0},
$$

then

$$
\int_{B_{r}(0)} u_{0 k} \geq M_{m}(r) \quad \text { for all } r \in[0, R] \text { and any } k \geq k_{0} .
$$

In fact, for any such $k$ we may use that $u_{0} \geq 0$ and proceed in a way quite similar to that in part (i) to estimate

$$
f_{B_{r}(0)} u_{0 k} \geq \frac{m}{\omega_{n} r^{n}} \int_{0}^{\frac{r}{r_{k}}} \sigma^{n-1} \zeta(\sigma) d \sigma \quad \text { for all } r \in(0, R),
$$

whence by (4.16),

$$
f_{B_{r}(0)} u_{0 k} \geq \frac{c_{4} m}{\omega_{n} r^{n}} \int_{0}^{\frac{r}{r_{k}}} \sigma^{n-1} d \sigma=\frac{c_{4} m}{n \omega_{n} r_{k}^{n}} \geq \frac{M_{m}(r)}{\left|B_{r}(0)\right|} \quad \text { for all } r \in\left(0, \frac{r_{k}}{2}\right]
$$

and

$$
f_{B_{r}(0)} u_{0 k} \geq \frac{m}{\omega_{n} r^{n}} \int_{0}^{\frac{1}{2}} \sigma^{n-1} \zeta(\sigma) d \sigma \geq \frac{c_{4} m}{2^{n} n \omega_{n} r^{n}} \geq \frac{c_{4} m}{2^{n} n \omega_{n} r_{k}^{n}} \geq \frac{M_{m}(r)}{\left|B_{r}(0)\right|}
$$

for all $r \in\left(\frac{r_{k}}{2}, r_{k}\right)$. As moreover

$$
\int_{B_{r}(0)} u_{0 k} \geq m \geq M_{m}(r) \quad \text { for all } r \in\left[r_{k}, R\right]
$$

due to the monotonicity of $M_{m}$ and the fact that $\int_{0}^{t} \sigma^{n-1} \zeta(\sigma) d \sigma=1$, we thus infer that (4.17) indeed holds. 


\section{Appendix: A comparison lemma}

An ingredient essential to our argument is the following variant of the parabolic comparison principle. Since we could not find an appropriate reference precisely covering the present situation, especially involving the present particular type of degenerate diffusion and nonsmooth comparison functions, we include a proof for completeness.

Lemma 5.1. Let $L>0, T>0, G:=(0, L) \times(0, T) \times \mathbb{R} \times(0, \infty) \times \mathbb{R}$, and $\phi \in C^{1}(G)$ be such that

$$
\frac{\partial \phi}{\partial y_{2}}\left(s, t, y_{0}, y_{1}, y_{2}\right) \geq 0 \quad \text { for all }\left(s, t, y_{0}, y_{1}, y_{2}\right) \in G,
$$

that for all $T_{0} \in(0, T)$ and $\Lambda>0$ there exists $C\left(T_{0}, \Lambda\right)>0$ fulfilling

$$
\left|\frac{\partial \phi}{\partial y_{0}}\left(s, t, y_{0}, y_{1}, y_{2}\right)\right| \leq C\left(T_{0}, \Lambda\right) \quad \text { for all }\left(s, t, y_{0}, y_{1}, y_{2}\right) \in G
$$

with $t \in\left(0, T_{0}\right)$ and $y_{1} \in(0, \Lambda)$, and such that for any $t_{0} \in(0, T)$ we have

$$
\frac{\partial \phi}{\partial y_{1}}(\cdot, t, \cdot, \cdot, \cdot) \in L_{\text {loc }}^{\infty}((0, L) \times \mathbb{R} \times(0, \infty) \times \mathbb{R}) .
$$

Suppose that $\underline{w}$ and $\bar{w}$ are two functions which belong to $C^{1}([0, L] \times[0, T))$ and satisfy

$$
\underline{w}_{s}(s, t)>0 \quad \text { and } \quad \bar{w}(s, t)>0 \quad \text { for all } s \in(0, L) \text { and } t \in(0, T)
$$

as well as

$$
\underline{w}(\cdot, t) \in W_{l o c}^{2, \infty}((0, L)) \quad \text { and } \quad \bar{w}(\cdot, t) \in W_{l o c}^{2, \infty}((0, L)) \quad \text { for all } t \in(0, T) .
$$

If moreover

$$
\underline{w}_{t} \leq \phi\left(s, t, \underline{w}, \underline{w}_{s}, \underline{w}_{s} s\right) \quad \text { and } \quad \bar{w}_{t} \geq \phi\left(s, t, \underline{w}, \underline{w}_{s}, \underline{w}_{s} s\right)
$$

for all $t \in(0, T)$ and a.e. $s \in(0, L)$, further

$$
\underline{w}(s, 0) \leq \bar{w}(s, 0) \quad \text { for all } s \in(0, L),
$$

as well as

$$
\underline{w}(0, t) \leq \bar{w}(0, t) \quad \text { and } \quad \underline{w}(L, t) \leq \bar{w}(L, t) \quad \text { for all } \quad t \in(0, T),
$$

then

$$
\underline{w}(s, t) \leq \bar{w}(s, t) \quad \text { for all } \quad s \in[0, L] \quad \text { and } \quad t \in[0, T) .
$$

Proof. We fix an arbitrary $T_{0} \in(0, T)$ and then obtain from (5.4) and the assumed regularity properties of $\underline{w}$ and $\bar{w}$ that there exists $\Lambda=\Lambda\left(T_{0}\right)>0$ such that

$0<\underline{w}_{s}(s, t)<\Lambda$ and $\quad 0<\bar{w}_{s}(s, t)<\Lambda \quad$ for all $s \in(0, L)$ and $t \in\left(0, T_{0}\right)$.

For $\varepsilon>0$, we then let $c_{5}:=C\left(T_{0}, \Lambda\right)$ with $C\left(T_{0}, \Lambda\right)>0$ as in (5.2), define

$$
z(s, t):=\underline{w}(s, t)-\bar{w}(s, t)-\varepsilon e^{2 c_{5} t} \quad \text { for } s \in[0, L] \text { and } t \in\left[0, T_{0}\right],
$$

and claim that

$$
z(s, t)<0 \quad \text { for all } s \in[0, L] \text { and } t \in\left[0, T_{0}\right)
$$


To verify this, supposing for contradiction that (5.12) is false, from (5.7) and (5.8) we would infer the existence of $s_{0} \in(0, L)$ and $t_{0} \in\left(0, T_{0}\right)$ such that

$$
\max _{(s, t) \in[0, L] \times\left[0, t_{0}\right]} z(s, t)=z\left(s_{0}, t_{0}\right)=0,
$$

in particular implying that

$$
z_{t}\left(s_{0}, t_{0}\right) \geq 0
$$

and

$$
z_{s}\left(s_{0}, t_{0}\right)=0 .
$$

Moreover, using (5.15) we obtain that $z\left(\cdot, t_{0}\right) \in W_{l o c}^{2, \infty}((0, L))$, so that we can find a null set $N \subset(0, L)$ such that $z_{s s}\left(s, t_{0}\right)$ exists for all $s \in(0, L) \backslash N$ and

$$
z_{s}\left(s, t_{0}\right)=\int_{s_{0}}^{s} z_{s s}\left(\sigma, t_{0}\right) d \sigma \quad \text { for all } s \in[0, L]
$$

according to (5.15), where for later use we note that enlarging $N$ if necessary we can furthermore achieve that both inequalities in (5.6) are valid at $\left(s, t_{0}\right)$ for all $s \in(0, L) \backslash N$. As $z\left(\cdot, t_{0}\right)$ attains its maximum at $s_{0}$ by (5.13), the identity (5.16) necessarily requires that there exists $\left(s_{j}\right)_{j \in \mathbb{N}} \subset\left(s_{0}, L\right) \backslash N$ such that $s_{j} \searrow s_{0}$ as $j \rightarrow \infty$ and

$$
z_{s s}\left(s_{j}, t_{0}\right) \leq 0 \quad \text { for all } j \in \mathbb{N},
$$

for otherwise (5.16) would imply that $z_{s}\left(s, t_{0}\right)>0$ for all $s \in\left(s_{0}, s_{\star}\right)$ with some $s_{\star} \in\left(s_{0}, L\right)$, which would clearly contradict (5.13).

Now differentiating (5.11), in view of (5.6) and our choice of $N$ we see that

$$
\begin{aligned}
z_{t} & =\underline{w}_{t}-\bar{w}_{t}-2 c_{5} \varepsilon e^{2 c_{5} t_{0}} \\
& \leq \phi\left(s, t_{0}, \underline{w}_{w}, \underline{w}_{s s}\right)-\phi\left(s, \underline{w}_{0}, \bar{w}_{s}, \bar{w}_{s}, \bar{w}_{s s}\right)-2 c_{5} \varepsilon e^{2 c_{5} t_{0}} \quad \text { for all } s \in N,
\end{aligned}
$$

so that from (5.17) we infer that

$$
\begin{aligned}
z_{t}\left(s_{j}, t_{0}\right) & \leq \phi\left(s_{j}, t_{0}, \underline{w}\left(s_{j}, t_{0}\right), \underline{w}_{s}\left(s_{j}, t_{0}\right), \bar{w}_{s s}\left(s_{j}, t_{0}\right)\right) \\
& -\phi\left(s_{j}, t_{0}, \bar{w}\left(s_{j}, t_{0}\right), \bar{w}_{s}\left(s_{j}, t_{0}\right), \bar{w}_{s s}\left(s_{j}, t_{0}\right)\right) \\
& -2 c_{5} \varepsilon e^{2 c_{5} \varepsilon t_{0}} \quad \text { for all } j \in \mathbb{N} .
\end{aligned}
$$

Here by the mean value theorem we have

$$
\begin{aligned}
\phi\left(s_{j},\right. & \left.t_{0}, \underline{w}\left(s_{j}, t_{0}\right), \underline{w}_{s}\left(s_{j}, t_{0}\right), \bar{w}_{s s}\left(s_{j}, t_{0}\right)\right) \\
& -\phi\left(s_{j}, t_{0}, \bar{w}\left(s_{j}, t_{0}\right), \bar{w}_{s}\left(s_{j}, t_{0}\right), \bar{w}_{s s}\left(s_{j}, t_{0}\right)\right) \\
& =\xi_{j} \cdot\left(\underline{w}\left(s_{j}, t_{0}\right)-\bar{w}\left(s_{j}, t_{0}\right)\right)+\lambda_{j} \cdot\left(\underline{w}_{s}\left(s_{j}, t_{0}\right)-\bar{w}_{s}\left(s_{j}, t_{0}\right)\right)
\end{aligned}
$$

with

$$
\begin{aligned}
\xi_{j}:= & \int_{0}^{1} \frac{\partial \phi}{\partial y_{0}}\left(s_{j}, t_{0}, \bar{w}\left(s_{j}, t_{0}\right)+\sigma\left(\underline{w}\left(s_{j}, t_{0}\right)-\bar{w}\left(s_{j}, t_{0}\right)\right), \bar{w}_{s}\left(s_{j}, t_{0}\right)\right. \\
& \left.+\sigma\left(\underline{w}_{s}\left(s_{j}, t_{0}\right)-\bar{w}_{s}\left(s_{j}, t_{0}\right)\right), \bar{w}_{s s}\left(s_{j}, t_{0}\right)\right) d \sigma
\end{aligned}
$$


and

$$
\begin{aligned}
\lambda_{j}:= & \int_{0}^{1} \frac{\partial \phi}{\partial y_{1}}\left(s_{j}, t_{0}, \bar{w}\left(s_{j}, t_{0}\right)+\sigma\left(\underline{w}\left(s_{j}, t_{0}\right)-\bar{w}\left(s_{j}, t_{0}\right)\right), \bar{w}_{s}\left(s_{j}, t_{0}\right)\right. \\
& \left.+\sigma\left(\underline{w}_{s}\left(s_{j}, t_{0}\right)-\bar{w}_{s}\left(s_{j}, t_{0}\right)\right), \bar{w}_{s s}\left(s_{j}, t_{0}\right)\right) d \sigma
\end{aligned}
$$

for $j \in \mathbb{N}$. Since $s_{j} \rightarrow s_{0}$ as $j \rightarrow \infty$, by continuity of $\underline{w}\left(\cdot, t_{0}\right)$ and $\bar{w}\left(\cdot, t_{0}\right)$ in $(0, L)$, by continuity and positivity of $\underline{w}_{s}\left(\cdot, t_{0}\right)$ and $\bar{w}_{s}\left(\cdot, t_{0}\right)$ in $(0, L)$, and by local boundedness of $\bar{w}_{s s}\left(\cdot, t_{0}\right)$ in $(0, L) \backslash N$ asserted by (5.5), we can find $\delta>0$ such that

$$
\begin{aligned}
& s_{j} \in[\delta, L-\delta], \quad \underline{w}\left(s_{j}, t_{0}\right) \in\left[-\frac{1}{\delta}, \frac{1}{\delta}\right], \quad \bar{w}\left(s_{j}, t_{0}\right) \in\left[-\frac{1}{\delta}, \frac{1}{\delta}\right], \\
& \underline{w}_{s}\left(s_{j}, t_{0}\right) \in\left[\delta, \frac{1}{\delta}\right], \quad \bar{w}_{s}\left(s_{j}, t_{0}\right) \in\left[\delta, \frac{1}{\delta}\right], \quad \text { and } \bar{w}_{s s}\left(s_{j}, t_{0}\right) \in\left[-\frac{1}{\delta}, \frac{1}{\delta}\right]
\end{aligned}
$$

for all $j \in \mathbb{N}$.

As a consequence of this and (5.3), there exists $c_{6}>0$ fulfilling

$$
\left|\eta_{j}\right| \leq c_{6} \quad \text { for all } j \in \mathbb{N} .
$$

Moreover, combining (5.10) with (5.2), by definition of $c_{5}$ we obtain that

$$
\left|\xi_{j}\right| \leq c_{5} \quad \text { for all } j \in \mathbb{N} .
$$

Collecting (5.19), (5.20), and (5.21) in (5.18) we can further estimate

$z_{t}\left(s_{j}, t_{0}\right) \leq c_{5} \cdot\left|\underline{w}\left(s_{j}, t_{0}\right)-\bar{w}\left(s_{j}, t_{0}\right)\right|+c_{6} \cdot\left|\underline{w}_{s}\left(s_{j}, t_{0}\right)-\bar{w}_{s}\left(s_{j}, t_{0}\right)\right|-2 c_{5} \varepsilon e^{2 c_{5} t_{0}}$

for all $j \in \mathbb{N}$.

Thanks to the fact that both $\underline{w}$ and $\bar{w}$ belong to $C^{1}\left((0, L) \times\left(0, T_{0}\right)\right)$, we may take $j \rightarrow \infty$ here to see that

$z_{t}\left(s_{0}, t_{0}\right) \leq c_{5} \cdot\left|\underline{w}\left(s_{0}, t_{0}\right)-\bar{w}\left(s_{0}, t_{0}\right)\right|+c_{6} \cdot\left|\underline{w}_{s}\left(s_{0}, t_{0}\right)-\bar{w}_{s}\left(s_{0}, t_{0}\right)\right|-2 c_{5} \varepsilon e^{2 c_{5} t_{0}}$.

Now observing that $\underline{w}\left(s_{0}, t_{0}\right)-\bar{w}\left(s_{0}, t_{0}\right)=\varepsilon e^{2 c_{5} t_{0}}$ by (5.13), and that $\underline{w}_{s}\left(s_{0}, t_{0}\right)-$ $\bar{w}_{s}\left(s_{0}, t_{0}\right)=0$ by (5.15), as a consequence of (5.14) we infer that

$$
0 \leq z_{t}\left(s_{0}, t_{0}\right) \leq c_{5} \varepsilon e^{2 c_{5} t_{0}}-2 c_{5} \varepsilon e^{2 c_{5} t_{0}}<0 .
$$

This absurd conclusion shows that actually (5.12) indeed holds, so that on letting $\varepsilon \searrow 0$ and then $T_{0} \nearrow T$ we end up with (5.9).

\section{ACKNOWLEDGEMENT}

The authors warmly thank the anonymous referee for numerous fruitful remarks which have led to a substantial improvement of this paper.

\section{REFERENCES}

[1] Fuensanta Andreu, Vicent Caselles, and José M. Mazón, The Cauchy problem for a strongly degenerate quasilinear equation, J. Eur. Math. Soc. (JEMS) 7 (2005), no. 3, 361-393, DOI 10.4171/JEMS/32. MR2156605

[2] Fuensanta Andreu, Vicent Caselles, José M. Mazón, and Salvador Moll, Finite propagation speed for limited flux diffusion equations, Arch. Ration. Mech. Anal. 182 (2006), no. 2, 269297, DOI 10.1007/s00205-006-0428-3. MR2259334

[3] Fuensanta Andreu, Vicent Caselles, José M. Mazón, Juan Soler, and Michela Verbeni, Radially symmetric solutions of a tempered diffusion equation. A porous media, flux-limited case, SIAM J. Math. Anal. 44 (2012), no. 2, 1019-1049, DOI 10.1137/110840297. MR2914259 
[4] Nicola Bellomo and Abdelghani Bellouquid, On multiscale models of pedestrian crowds from mesoscopic to macroscopic, Commun. Math. Sci. 13 (2015), no. 7, 1649-1664, DOI 10.4310/CMS.2015.v13.n7.a1. MR.3393169

[5] Nicola Bellomo, Abdelghani Bellouquid, and Damian Knopoff, From the microscale to collective crowd dynamics, Multiscale Model. Simul. 11 (2013), no. 3, 943-963, DOI 10.1137/130904569. MR 3105783

[6] Nicola Bellomo, Abdelghani Bellouquid, Juan Nieto, and Juan Soler, Multiscale biological tissue models and flux-limited chemotaxis for multicellular growing systems, Math. Models Methods Appl. Sci. 20 (2010), no. 7, 1179-1207, DOI 10.1142/S0218202510004568. MR2673415

[7] Nicola Bellomo, Abdelghani Bellouquid, Youshan Tao, and Michael Winkler, Toward a mathematical theory of Keller-Segel models of pattern formation in biological tissues, Math. Models Methods Appl. Sci. 25 (2015), no. 9, 1663-1763, DOI 10.1142/S021820251550044X. MR.3351175

[8] Nicola Bellomo and Michael Winkler, A degenerate chemotaxis system with flux limitation: Maximally extended solutions and absence of gradient blow-up, Comm. Partial Differential Equations 42 (2017), no. 3, 436-473, DOI 10.1080/03605302.2016.1277237. MR3620894

[9] Piotr Biler, Global solutions to some parabolic-elliptic systems of chemotaxis, Adv. Math. Sci. Appl. 9 (1999), no. 1, 347-359. MR 1690388

[10] Piotr Biler, Lucilla Corrias, and Jean Dolbeault, Large mass self-similar solutions of the parabolic-parabolic Keller-Segel model of chemotaxis, J. Math. Biol. 63 (2011), no. 1, 1-32, DOI 10.1007/s00285-010-0357-5. MR2806487

[11] Tobias Black, Sublinear signal production in a two-dimensional Keller-Segel-Stokes system, Nonlinear Anal. Real World Appl. 31 (2016), 593-609, DOI 10.1016/j.nonrwa.2016.03.008. MR3490860

[12] Vincent Calvez and José A. Carrillo, Volume effects in the Keller-Segel model: energy estimates preventing blow-up, J. Math. Pures Appl. (9) 86 (2006), no. 2, 155-175, DOI 10.1016/j.matpur.2006.04.002. MR2247456

[13] Juan Calvo, Juan Campos, Vicent Caselles, Óscar Sánchez, and Juan Soler, Flux-saturated porous media equations and applications, EMS Surv. Math. Sci. 2 (2015), no. 1, 131-218, DOI 10.4171/EMSS/11. MR3354957

[14] Juan Calvo, Juan Campos, Vicent Caselles, Óscar Sánchez, and Juan Soler, Pattern formation in a flux limited reaction-diffusion equation of porous media type, Invent. Math. 206 (2016), no. 1, 57-108, DOI 10.1007/s00222-016-0649-5. MR3556525

[15] Juan Calvo, Juan Campos, Vicent Caselles, Óscar Sánchez, and Juan Soler, Qualitative behaviour for flux-saturated mechanisms: travelling waves, waiting time and smoothing effects, J. Eur. Math. Soc. (JEMS) 19 (2017), no. 2, 441-472, DOI 10.4171/JEMS/670. MR3605021

[16] Juan Campos, Pilar Guerrero, Óscar Sánchez, and Juan Soler, On the analysis of traveling waves to a nonlinear flux limited reaction-diffusion equation, Ann. Inst. H. Poincaré Anal. Non Linéaire 30 (2013), no. 1, 141-155, DOI 10.1016/j.anihpc.2012.07.001. MR.3011295

[17] Xinru Cao, Global bounded solutions of the higher-dimensional Keller-Segel system under smallness conditions in optimal spaces, Discrete Contin. Dyn. Syst. 35 (2015), no. 5, 18911904, DOI 10.3934/dcds.2015.35.1891. MR 3294230

[18] Carlo Cercignani, Reinhard Illner, and Mario Pulvirenti, The mathematical theory of dilute gases, Applied Mathematical Sciences, vol. 106, Springer-Verlag, New York, 1994. MR.1307620

[19] Tomasz Cieślak and Philippe Laurençot, Finite time blow-up for a one-dimensional quasilinear parabolic-parabolic chemotaxis system, Ann. Inst. H. Poincaré Anal. Non Linéaire 27 (2010), no. 1, 437-446, DOI 10.1016/j.anihpc.2009.11.016. MR2580517

[20] Tomasz Cieślak and Christian Stinner, Finite-time blowup and global-in-time unbounded solutions to a parabolic-parabolic quasilinear Keller-Segel system in higher dimensions, J. Differential Equations 252 (2012), no. 10, 5832-5851, DOI 10.1016/j.jde.2012.01.045. MR2902137

[21] Tomasz Cieślak and Christian Stinner, Finite-time blowup in a supercritical quasilinear parabolic-parabolic Keller-Segel system in dimension 2, Acta Appl. Math. 129 (2014), 135146, DOI 10.1007/s10440-013-9832-5. MR3152080

[22] Tomasz Cieślak and Christian Stinner, New critical exponents in a fully parabolic quasilinear Keller-Segel system and applications to volume filling models, J. Differential Equations 258 (2015), no. 6, 2080-2113, DOI 10.1016/j.jde.2014.12.004. MR3302531 
[23] Michael Winkler and Kianhwa C. Djie, Boundedness and finite-time collapse in a chemotaxis system with volume-filling effect, Nonlinear Anal. 72 (2010), no. 2, 1044-1064, DOI 10.1016/j.na.2009.07.045. MR2579368

[24] Miguel A. Herrero and Juan J. L. Velázquez, A blow-up mechanism for a chemotaxis model, Ann. Scuola Norm. Sup. Pisa Cl. Sci. (4) 24 (1997), no. 4, 633-683 (1998). MR.1627338

[25] Tomas Hillen and Kevin J. Painter, A user's guide to PDE models for chemotaxis, J. Math. Biol. 58 (2009), no. 1-2, 183-217, DOI 10.1007/s00285-008-0201-3. MR2448428

[26] Dirk Horstmann, From 1970 until present: the Keller-Segel model in chemotaxis and its consequences. I, Jahresber. Deutsch. Math.-Verein. 105 (2003), no. 3, 103-165. MR2013508

[27] Dirk Horstmann and Michael Winkler, Boundedness vs. blow-up in a chemotaxis system, J. Differential Equations 215 (2005), no. 1, 52-107, DOI 10.1016/j.jde.2004.10.022. MR2146345

[28] Sachiko Ishida, Kiyotaka Seki, and Tomomi Yokota, Boundedness in quasilinear Keller-Segel systems of parabolic-parabolic type on non-convex bounded domains, J. Differential Equations 256 (2014), no. 8, 2993-3010, DOI 10.1016/j.jde.2014.01.028. MR3199754

[29] Willi Jäger and Stephan Luckhaus, On explosions of solutions to a system of partial differential equations modelling chemotaxis, Trans. Amer. Math. Soc. 329 (1992), no. 2, 819-824, DOI 10.2307/2153966. MR 1046835

[30] Evelin F. Keller and Lee A. Segel, Initiation of slime mold aggregation viewed as an instability, J. Theor. Biol. 26 (1970), 399-415.

[31] Alexander Kiselev and Xiaoqian Xu, Suppression of chemotactic explosion by mixing, Arch. Ration. Mech. Anal. 222 (2016), no. 2, 1077-1112, DOI 10.1007/s00205-016-1017-8. MR.3544323

[32] Remigiusz Kowalczyk and Zuzanna Szymańska, On the global existence of solutions to an aggregation model, J. Math. Anal. Appl. 343 (2008), no. 1, 379-398, DOI 10.1016/j.jmaa.2008.01.005. MR2412135

[33] Dong-mei Liu and You-shan Tao, Boundedness in a chemotaxis system with nonlinear signal production, Appl. Math. J. Chinese Univ. Ser. B 31 (2016), no. 4, 379-388, DOI 10.1007/s11766-016-3386-z. MR3579264

[34] Noriko Mizoguchi and Michael Winkler, Blow-up in the two-dimensional parabolic KellerSegel system, preprint.

[35] Toshitaka Nagai, Blowup of nonradial solutions to parabolic-elliptic systems modeling chemotaxis in two-dimensional domains, J. Inequal. Appl. 6 (2001), no. 1, 37-55, DOI 10.1155/S1025583401000042. MR.1887324

[36] Toshitaka Nagai, Takasi Senba, and Kiyoshi Yoshida, Application of the Trudinger-Moser inequality to a parabolic system of chemotaxis, Funkcial. Ekvac. 40 (1997), no. 3, 411-433. MR.1610709

[37] Koichi Osaki, Tohru Tsujikawa, Atsushi Yagi, and Masayasu Mimura, Exponential attractor for a chemotaxis-growth system of equations, Nonlinear Anal. 51 (2002), no. 1, Ser. A: Theory Methods, 119-144, DOI 10.1016/S0362-546X(01)00815-X. MR1915744

[38] Koichi Osaki and Atsushi Yagi, Finite dimensional attractor for one-dimensional Keller-Segel equations, Funkcial. Ekvac. 44 (2001), no. 3, 441-469. MR.1893940

[39] Takasi Senba and Takasi Suzuki, A quasi-linear parabolic system of chemotaxis, Abstr. Appl. Anal., posted on 2006, Art. ID 23061, 21, DOI 10.1155/AAA/2006/23061. MR2211660

[40] Youshan Tao and Michael Winkler, Boundedness in a quasilinear parabolic-parabolic KellerSegel system with subcritical sensitivity, J. Differential Equations 252 (2012), no. 1, 692-715, DOI 10.1016/j.jde.2011.08.019. MR2852223

[41] M. Verbeni, O. Sánchez, E. Mollica, I. Siegl-Cachedenier, A. Carleton, A. Guerrero, A. Ruiz i Atalba, and J. Soler, Morphogenetic action through flux-limited spreading, Physics of Life Reviews 10 (2013), 457-475.

[42] Michael Winkler, Aggregation vs. global diffusive behavior in the higher-dimensional Keller-Segel model, J. Differential Equations 248 (2010), no. 12, 2889-2905, DOI 10.1016/j.jde.2010.02.008. MR2644137

[43] Michael Winkler, Boundedness in the higher-dimensional parabolic-parabolic chemotaxis system with logistic source, Comm. Partial Differential Equations 35 (2010), no. 8, 1516-1537, DOI 10.1080/03605300903473426. MR2754053

[44] Michael Winkler, Does a 'volume-filling effect' always prevent chemotactic collapse?, Math. Methods Appl. Sci. 33 (2010), no. 1, 12-24, DOI 10.1002/mma.1146. MR2591220 
[45] Michael Winkler, Finite-time blow-up in the higher-dimensional parabolic-parabolic KellerSegel system (English, with English and French summaries), J. Math. Pures Appl. (9) 100 (2013), no. 5, 748-767, DOI 10.1016/j.matpur.2013.01.020. MR.3115832

[46] Dariusz Wrzosek, Long-time behaviour of solutions to a chemotaxis model with volumefilling effect, Proc. Roy. Soc. Edinburgh Sect. A 136 (2006), no. 2, 431-444, DOI 10.1017/S0308210500004649. MR2218162

Department of Mathematics, Faculty of Sciences, King Abdulaziz University, JedDAH, SAUdi ARABIA

Current address: Politecnico of Torino, 10129 Torino, Italy

E-mail address: nicola.bellomo@polito.it

Institut für Mathematik, Universität Paderborn, 33098 Paderborn, Germany

E-mail address: michael.winkler@math.uni-paderborn.de 